\title{
Contributions of vacancies and self-interstitials to self-diffusion in silicon under thermal equilibrium and nonequilibrium conditions
}

\author{
R. Kube and H. Bracht* \\ Institute of Materials Physics, Westfälische Wilhelms-Universität Münster, D-48149 Münster, Germany \\ E. Hüger and H. Schmidt \\ Institut für Metallurgie, AG Mikrokinetik, Technische Universität Clausthal, D-38678 Clausthal-Zellerfeld, Germany \\ J. Lundsgaard Hansen and A. Nylandsted Larsen \\ Department of Physics and Astronomy, University of Aarhus, DK-8000 Aarhus, Denmark \\ J. W. Ager III and E. E. Haller \\ Lawrence Berkeley National Laboratory and University of California, Berkeley, California 94720, USA \\ T. Geue and J. Stahn \\ Laboratory for Neutron Scattering, Paul Scherrer Institut, Villigen PSI, CH-5232, Switzerland
}

(Received 24 May 2013; published 15 August 2013)

\begin{abstract}
Since many years, the contribution of vacancies $(V)$ and self-interstitials $(I)$ to silicon ( $\mathrm{Si}$ ) self-diffusion is a matter of debate. Native defects and their interaction among themselves and with foreign atoms influence the processes taking place during device fabrication, starting with the growth of Si single crystals and ending with doping of nanosized electronic devices. Considering this relevance, it is remarkable that present data about the properties of native point defects in $\mathrm{Si}$ are still limited and controversial. This work reports experiments on self-diffusion in Si for temperatures between $650^{\circ} \mathrm{C}$ and $960^{\circ} \mathrm{C}$ to verify recent results of Shimizu et al. [Phys. Rev. Lett. 98, 095901 (2007)] that give rise to inconsistencies in $V$-mediated self- and dopant diffusion. Two different structures of isotopically controlled epitaxial layers of $\mathrm{Si}$ are used for the diffusion study. One structure consisting of 20 bilayers of ${ }^{29} \mathrm{Si} /{ }^{28} \mathrm{Si}$ was grown by molecular beam epitaxy (MBE). The other structure with a ${ }^{28} \mathrm{Si}$ layer sandwiched between natural $\mathrm{Si}$ was grown by means of chemical vapor deposition. Self-diffusion in $\left({ }^{29} \mathrm{Si} /{ }^{28} \mathrm{Si}\right)_{20}$ multilayers (ML) was analyzed by means of secondary ion mass spectrometry (SIMS) and neutron reflectometry, whereas self-diffusion in ${ }^{\text {nat }} \mathrm{Si} /{ }^{28} \mathrm{Si} /{ }^{\text {nat }} \mathrm{Si}$ sandwich $(\mathrm{SW})$ structures was measured with SIMS only. Analysis of the experimental profiles reveals an enhanced self-diffusion in ML compared to SW structures. The enhanced diffusion is ascribed to the dissolution of $V$ - and $I$-related defect clusters grown-in during MBE. On the other hand, self-diffusion in the SW structures accurately confirms the data of Shimizu et al. that are considered to represent data for thermal equilibrium conditions. The temperature dependence of self-diffusion is described by $V$ - and $I$-mediated contributions with temperature-dependent thermodynamic properties of $V$. This interpretation can solve the inconsistency between self- and dopant diffusion in $\mathrm{Si}$, but further experiments are required to verify this concept.
\end{abstract}

DOI: 10.1103/PhysRevB.88.085206

PACS number(s): 61.72.uf, 61.72.jd, 61.72.jj, 66.30.H-

\section{INTRODUCTION}

Self-diffusion in Si is a subject of a long-standing debate that starts with the first experiments performed by Peart, ${ }^{1}$ Masters and Fairfield, ${ }^{2,3}$ and Ghoshtagore ${ }^{4}$ almost 50 years ago. Earlier experiments reveal a $1-\mathrm{eV}$ spread in the value of the activation enthalpy $Q$ of self-diffusion that represents the sum of the formation $H^{F}$ and migration $H^{M}$ enthalpy of the native defect mediating the diffusion process (see Ref. 5 and references therein). This spread was considered to indicate a change in the dominant mechanisms of self-diffusion although a direct proof was difficult because previous samples and analysis methods have hampered a study of self-diffusion over a wide range of temperatures (see Ref. 5). The availability of isotopically enriched $\mathrm{Si}$ and the improvements in epitaxial deposition techniques such as molecular beam epitaxy (MBE) and chemical vapor deposition (CVD) made the preparation of $\mathrm{Si}$ isotope structures possible. By means of isotopically controlled Si samples, the self-diffusion could be investigated over a wide range of temperatures. ${ }^{5-8}$ For temperatures between $855^{\circ} \mathrm{C}$ and $1388^{\circ} \mathrm{C}$, the self-diffusion is accurately described by one single diffusion activation enthalpy $Q$ of $4.75 \mathrm{eV}^{5}{ }^{5}$ The close agreement of the self-diffusion data to the self-interstitial $(I)$ contribution $D_{\mathrm{Si}}^{I}$ to $\mathrm{Si}$ self-diffusion deduced from metal-diffusion experiments demonstrate that $I$ dominate self-diffusion at temperatures above $900^{\circ} \mathrm{C} .{ }^{5,9}$ Following this successful application of $\mathrm{Si}$ isotope structures for self-diffusion studies, the interference between self- and dopant diffusion ${ }^{10,11}$ and the impact of homogeneous background doping on self-diffusion was investigated. ${ }^{12-15}$ These studies yield details about the charge state of the native point defects mediating self-diffusion (see Ref. 11). Although general consensus exists on the magnitude of self-diffusion under electronically intrinsic conditions and the impact of doping, the contribution $D_{\mathrm{Si}}^{V}$ of vacancies $(V)$ to self-diffusion still remains unsolved. This, in particular, becomes evident by 
the spread in the data reported for the activation enthalpy of self-diffusion via $V$ that ranges from 3.6 to $4.9 \mathrm{eV}^{5,6,8}$ The discussion on the contribution of $D_{\mathrm{Si}}^{V}$ to self-diffusion shows the need for additional self-diffusion experiments at temperatures below $850{ }^{\circ} \mathrm{C}$ and for calculations on the correlation factor $f_{I}$ of self-diffusion via $I$. Whereas the latter aspect accounts for only $20 \%-30 \%$ variations on the contribution $D_{\mathrm{Si}}^{I}$ to self-diffusion, ${ }^{16}$ the former demand for self-diffusion studies at low temperatures would increase the sensitivity in the detection of additional native defects that beside $I$ mediate self-diffusion. Recently, Si self-diffusion has been explored for temperatures between $875^{\circ} \mathrm{C}$ and $735^{\circ} \mathrm{C}$ utilizing a periodic isotope superlattice (SL) with 20 bilayers of ${ }^{28} \mathrm{Si} /{ }^{30} \mathrm{Si}^{8}$ The diffusion-induced intermixing of the isotope structure was determined from the annealing time dependence of the Raman shift of the longitudinal optical phonon frequencies of the isotope SL. For temperatures above $850{ }^{\circ} \mathrm{C}$, literature data of self-diffusion are accurately reproduced. ${ }^{8}$ With decreasing temperature, a clear deviation of self-diffusion from the data expected from high-temperature extrapolations is observed. Taking into account the successful extension of self-diffusion data to lower temperatures and the results of metal diffusion on the contribution of $I$ to self-diffusion, Shimizu et al. ${ }^{8}$ determined an activation enthalpy of $Q_{V}=3.6 \mathrm{eV}$ for the $V$ contribution $D_{\mathrm{Si}}^{V}$ to self-diffusion. Although the result reported by Shimizu et al. seems, on first sight, to be consistent with the properties of $V$ at cryogenic temperatures, ${ }^{17,18}$ the value of $3.6 \mathrm{eV}$ is at variance with experiments on $V$-mediated dopant diffusion in Si that rather suggest an activation enthalpy above $4 \mathrm{eV}$ for self-diffusion via $V .{ }^{19}$ In order to unravel the present situation, first the experimental results of Shimizu et al. ${ }^{8}$ must be verified and second a diffusion model needs to be developed that is consistent with both self- and dopant diffusion in $\mathrm{Si}$ and combines the properties of $V$ determined for cryogenic temperatures. Since self-atom mixing at low temperatures will suffer from small diffusion lengths, appropriate techniques must be applied to determine the diffusion-induced broadening of a $\mathrm{Si}$ isotope structure annealed at temperatures below $850{ }^{\circ} \mathrm{C}$.

In the following experimental results on self-diffusion in $\left({ }^{29} \mathrm{Si} /{ }^{28} \mathrm{Si}\right)_{20}$ multilayers $(\mathrm{ML})$ and ${ }^{\text {nat }} \mathrm{Si} /{ }^{28} \mathrm{Si} /{ }^{\text {nat }} \mathrm{Si}$ sandwich (SW) structures analyzed by means of secondary ion mass spectrometry (SIMS) and neutron reflectometry (NR) are presented. Self-diffusion data obtained from SW structures are in accurate agreement with recent results of Shimizu et al. ${ }^{8}$ In contrast, self-diffusion in isotope ML is clearly enhanced. A diffusion model is proposed that describes the temperature dependence of self-diffusion under thermal equilibrium conditions and the enhanced self-diffusion consistently. The contributions of $D_{\mathrm{Si}}^{V}$ and $D_{\mathrm{Si}}^{I}$ to self-diffusion and the kinetic of $V$ - and $I$-related defect clusters determined on the basis of this model are consistent with dopant diffusion in $\mathrm{Si}$ and the stability of defect clusters formed by $V$ and $I$.

\section{EXPERIMENT}

For the self-diffusion experiments, we used two different isotopically controlled Si structures. Structure No. 1 consists of 20 bilayers of undoped ${ }^{29} \mathrm{Si} /{ }^{28} \mathrm{Si}$ grown by means of $\mathrm{MBE}$ at $750^{\circ} \mathrm{C}$ on top of a 270 -nm-thick natural Si buffer
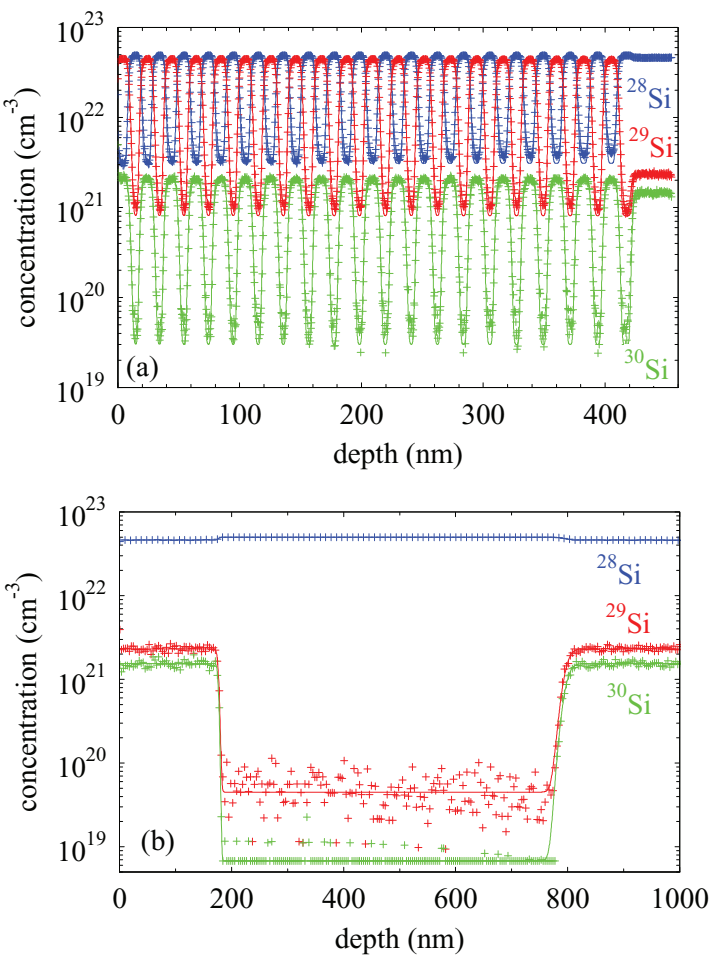

FIG. 1. (Color online) (a) SIMS concentration profiles of ${ }^{28} \mathrm{Si}$, ${ }^{29} \mathrm{Si}$, and ${ }^{30} \mathrm{Si}$ of the 20 -bilayer ${ }^{29} \mathrm{Si} /{ }^{28} \mathrm{Si}$ epitaxial structure 1 grown by means of MBE on a natural Si substrate wafer. The multilayer (ML) structure was used for self-diffusion experiments at temperatures between $650{ }^{\circ} \mathrm{C}$ and $960{ }^{\circ} \mathrm{C}$. The diffusional broadening of the isotope structure was analyzed both with SIMS and NR. (b) SIMS concentration profiles of ${ }^{28} \mathrm{Si},{ }^{29} \mathrm{Si}$, and ${ }^{30} \mathrm{Si}$ of the ${ }^{\text {nat }} \mathrm{Si} /{ }^{28} \mathrm{Si} /{ }^{\text {nat }} \mathrm{Si}$ epitaxial sandwich (SW) structure 2 grown by means of CVD on a natural $\mathrm{Si}$ substrate. The sandwich structure was utilized for selfdiffusion experiments at temperatures between $750{ }^{\circ} \mathrm{C}$ and $900{ }^{\circ} \mathrm{C}$. The diffusional broadening at the near-surface ${ }^{\text {nat }} \mathrm{Si} /{ }^{28} \mathrm{Si}$ interface was analyzed with SIMS. The solid lines in (a) and (b) represent best fits based on the Eq. (2) to the as-grown Si profiles. The convolution integral (2) takes sputter broadening effects into account.

layer. The layer structure was deposited on a (100)-oriented single-crystalline $\mathrm{Si}$ substrate wafer with a boron (B) doping level of $2 \times 10^{15} \mathrm{~cm}^{-3}$. The thickness of the individual ${ }^{29} \mathrm{Si}$ and ${ }^{28} \mathrm{Si}$ layers was determined with SIMS and NR to $\sim 10.5 \mathrm{~nm}$. This adds up to a total thickness of the isotope stack of $\sim 420 \mathrm{~nm}$. A SIMS analysis of the as-grown $\left({ }^{29} \mathrm{Si} /{ }^{28} \mathrm{Si}\right)_{20}$ ML structure 1 that illustrates the distribution of the stable $\mathrm{Si}$ isotopes is shown in Fig. 1(a). The oxygen and carbon concentration in the epitaxial layer measured with SIMS is $\sim 10^{16} \mathrm{~cm}^{-3}$ and $3 \times 10^{18}$, respectively. Structure No. 2 consists of a $\sim 600$-nm-thick undoped isotopically enriched ${ }^{28} \mathrm{Si}$ layer sandwiched between a buffer and cap layer of ${ }^{\text {nat }} \mathrm{Si}$. The buffer and cap layer are each about $200 \mathrm{~nm}$ thick. This layer structure was grown by chemical vapor deposition (CVD) at $800^{\circ} \mathrm{C}$ on a B-doped (100)-oriented Si substrate with a B doping of $2 \times 10^{15} \mathrm{~cm}^{-3}$. Figure 1(b) illustrates the distribution of the $\mathrm{Si}$ isotopes in the as-grown SW structure 2. The carbon and oxygen concentration of structure 2 was measured with SIMS to $5 \times 10^{17}$ and $<10^{18} \mathrm{~cm}^{-3}$. This structure was already 


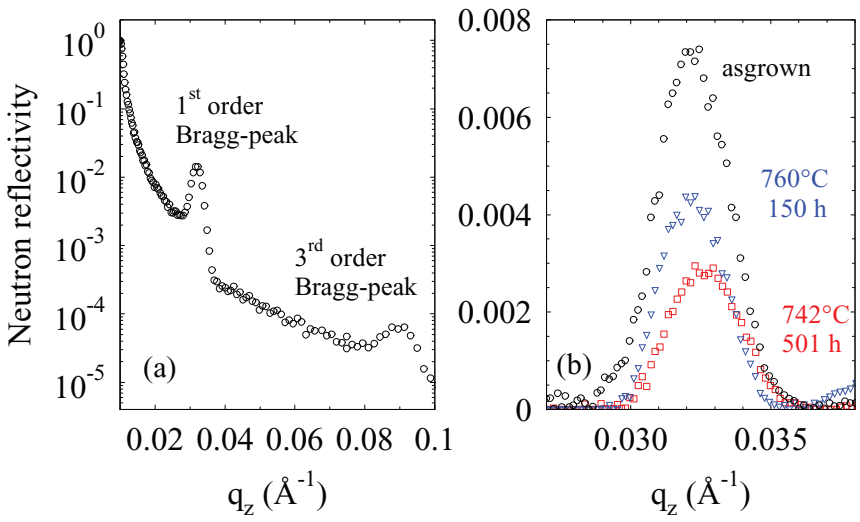

FIG. 2. (Color online) (a) Neutron reflectivity pattern of an asgrown $\left({ }^{29} \mathrm{Si} /{ }^{28} \mathrm{Si}\right)_{20}$ isotope multilayer. (b) First-order Bragg peak of the isotope multilayer after annealing at the temperatures and times indicated in comparison to the NR pattern of an as-grown sample. The decrease in the Bragg peak due to annealing is a measure of the self-diffusion coefficient. A background correction was applied to the Bragg peak.

used in previous self-diffusion studies and marked as No. 5 (see Refs. 20 and 21).

Samples with lateral dimensions of $5 \times 5 \mathrm{~mm}^{2}$ and $10 \times$ $10 \mathrm{~mm}^{2}$ were cut from the deposited wafers, cleaned in organic solvents, etched in 5\% diluted HF, and purged in distilled and deionized water. The smaller samples were used for diffusion experiments, which were analyzed with SIMS, and the larger samples were used for both SIMS and NR studies. The samples were sealed in quartz ampoules under pure argon (99.999\%) that were evacuated to a pressure of $<10^{-6}$ mbar beforehand. Diffusion experiments were performed in resistance furnaces. The temperature was monitored with an accuracy of $\pm 2 \mathrm{~K}$ with a calibrated $\mathrm{Pt} / \mathrm{PtRh}$ thermocouple contacting the ampoule. After annealing, the distribution of the Si isotopes in structures 1 and 2 was measured with SIMS. In addition, the diffusional broadening of structure 1 was analyzed with NR. The isotopically modulated $\left({ }^{29} \mathrm{Si} /{ }^{28} \mathrm{Si}\right)_{20} \mathrm{ML}$ is well suited for NR due to the difference in the neutron scattering lengths of ${ }^{29} \mathrm{Si}(4.7 \mathrm{fm})$ and ${ }^{28} \mathrm{Si}(4.107 \mathrm{fm})$. Details are given in Ref. 22. A typical neutron reflectivity pattern of the as-grown ML is shown in Fig. 2(a). The first- and third-order Bragg peaks of the periodic ${ }^{29} \mathrm{Si} /{ }^{28} \mathrm{Si}$ ML stack are clearly resolved. Annealing of the isotope structure leads to an intermixing at the interfaces. The intermixing of structure 1 in comparison to the as-grown sample is detected by NR in the decrease of the first-order Bragg peak or by SIMS analyses in the increased broadening of the ${ }^{29} \mathrm{Si} /{ }^{28} \mathrm{Si}$ interfaces. Figures 2 (b) and 3(a) demonstrate the decrease in the Bragg peak and the diffusional broadening, respectively, of the ML structure measured after annealing at the temperatures and times indicated. Self-diffusion experiments with the SW structure 2 were analyzed with SIMS only. Typical profiles obtained after diffusion annealing at $750{ }^{\circ} \mathrm{C}$ and $763^{\circ} \mathrm{C}$ are shown in Fig. 3(b).

A TOF-SIMS 5 system at TASCON GmbH Münster operating in a dual beam mode was used for SIMS profiling of the $\mathrm{Si}$ isotopes in structures 1 and 2. Bismuth ions with an energy of $25 \mathrm{keV}$ served as analysis beam and oxygen
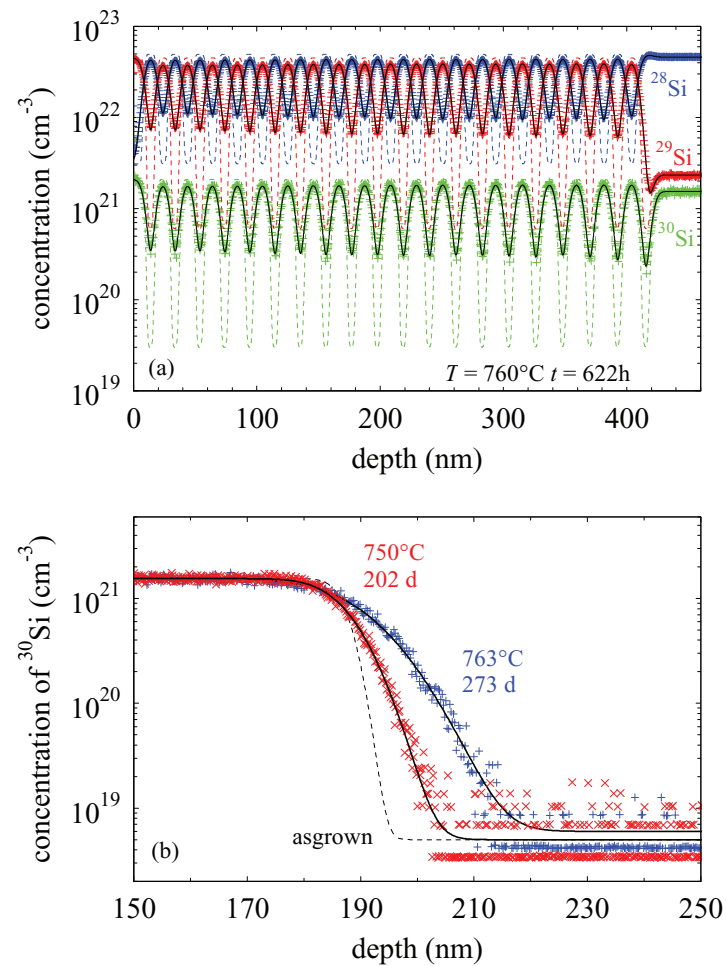

FIG. 3. (Color online) (a) Concentration profiles (symbols) of ${ }^{28} \mathrm{Si},{ }^{29} \mathrm{Si}$, and ${ }^{30} \mathrm{Si}$ of the $\left({ }^{29} \mathrm{Si} /{ }^{28} \mathrm{Si}\right)_{20}$ multilayer 1 measured with SIMS after annealing at $760{ }^{\circ} \mathrm{C}$ for $622 \mathrm{~h}$ in comparison to the as-grown $\mathrm{Si}$ profiles (dashed lines). Solid lines represent best fits to the experimental ${ }^{28} \mathrm{Si},{ }^{29} \mathrm{Si}$, and ${ }^{30} \mathrm{Si}$ profiles based on Eqs. (2), (3), and (5). (b) Concentration profiles (symbols) of ${ }^{30} \mathrm{Si}$ of the ${ }^{\text {nat }} \mathrm{Si} /{ }^{28} \mathrm{Si} /{ }^{\text {nat }} \mathrm{Si}$ isotope structure 2 measured with SIMS after annealing at the temperatures and times indicated in comparison to the as-grown ${ }^{30} \mathrm{Si}$ profile (dashed line). Solid lines represent best fits based on Eqs. (2), (4), and (5).

ions with an energy of $1 \mathrm{keV}(2 \mathrm{keV})$ as sputter beam for profiling the $\mathrm{Si}$ isotopes in structure 1 (2). Oxygen and carbon concentrations were determined by means of a CAMECA IMS4f system at RTG Mikroanalyse $\mathrm{GmbH}$ Berlin using 14.5-keV cesium as primary ion. The depths of the craters left by the TOF-SIMS and SIMS analyses were determined with an optical profilometer and a Talystep profilometer, respectively, to an accuracy of about $10 \%$. Neutron reflectometry was performed at the Swiss spallation neutron source (SINQ). The time-of-flight reflectometer AMOR was used at incoming neutron wavelengths between 0.2 and $0.9 \mathrm{~nm}$. Reflectivity patterns were measured at incident angles between $0.2^{\circ}$ and $3.0^{\circ}$. The resolution of the reflectometer was about $\Delta q_{z} / q_{z}=$ $5 \%$. Further details of the method are given in Refs. 23 and 24.

\section{RESULTS AND DISCUSSION}

Figures 2(b) and 3(a) demonstrate the neutron reflectivity pattern and the diffusional broadening of the isotope structure 1 measured, respectively, with NR and SIMS after annealing. Both techniques provide information about the self-diffusion coefficient $D_{\mathrm{Si}}$. In the case of the NR study, $D_{\mathrm{Si}}$ is determined 
from the background corrected Bragg peak by means of

$$
I(t)=I_{0} \exp \left(-\frac{8 \pi^{2} n^{2} D_{\mathrm{Si}}}{l^{2}} t\right),
$$

where $I(t)\left(I_{0}\right)$ is the integrated intensity of the Bragg peak of the annealed (as-grown) ML structure. $n, l$, and $t$ denote the order of the Bragg peak, the bilayer thickness, and diffusion time, respectively. The integrated intensity $I(t)$ is obtained by the integral of a Gaussian function fitted to the Bragg peak. Diffusivities can also be extracted by fitting the complete reflectivity pattern using an adequate simulation tool like the program PARRATT32. ${ }^{25,26}$ The same results are obtained within error limits. On the other hand, the diffusional broadening of the ML and SW structures is described by means of the following convolution integral

$$
C_{\mathrm{Si}}(x, t)=\int_{-\infty}^{\infty} C^{*}\left(x^{\prime}\right) g\left(x-x^{\prime}\right) d x^{\prime}
$$

that takes instrumental broadening effects due to sputter profiling into account. Here, $C_{\mathrm{Si}}, C^{*}$, and $g$ are the measured $\mathrm{Si}$ profile, the true profile, and the resolution function, respectively. The true profile of the ML structure is considered to be described by

$$
\begin{aligned}
C^{*}= & \frac{C_{1}+C_{3}}{2}+\frac{C_{1}-C_{2}}{2} \sum_{i=1}^{39}(-1)^{i} \operatorname{erf}\left(\frac{x-x_{i}}{r_{i}}\right) \\
& +\frac{C_{3}-C_{2}}{2} \operatorname{erf}\left(\frac{x-x_{40}}{r_{40}}\right) .
\end{aligned}
$$

This equation represents the solution of Fick's law for selfdiffusion of ${ }^{28} \mathrm{Si},{ }^{29} \mathrm{Si}$, and ${ }^{30} \mathrm{Si}$ across the isotope interfaces located at $x_{i} . C_{2}$ is the concentration of the respective $\mathrm{Si}$ isotope in the enriched ${ }^{28} \mathrm{Si}$ layer, $C_{1}$ the concentration to the left and right sides of this layer, and $C_{3}$ the concentration of the $\mathrm{Si}$ isotopes in the natural buffer layer. The diffusional broadening at the interface $x_{i}$ is described by the parameter $r_{i}=2 \sqrt{D_{\mathrm{Si}} t}$. In the case of the $\mathrm{SW}$ isotope structure, the true profile is described by

$$
C^{*}=\frac{C_{1}+C_{2}}{2}+\frac{C_{2}-C_{1}}{2} \operatorname{erf}\left(\frac{x-d}{r}\right),
$$

where $C_{1}$ and $C_{2}$ are the concentrations of ${ }^{30} \mathrm{Si}$ in the natural $\mathrm{Si}$ cap layer and the ${ }^{28} \mathrm{Si}$ isotope layer, respectively, and $r=$ $2 \sqrt{D_{\mathrm{Si}} t}$. A Gaussian function

$$
g(x)=\frac{1}{\sigma \sqrt{2 \pi}} \exp \left(-\frac{x^{2}}{2 \sigma^{2}}\right)
$$

with the standard deviation $\sigma$ was considered as resolution function in Eq. (2) that fulfills the condition

$$
\int_{-\infty}^{\infty} g(x) d x=1 .
$$

The resolution functions $g(x)$, i.e., $\sigma$ for structures 1 and 2 , were determined by best fits based on Eq. (2) to the experimental as-grown profiles assuming an interfacial broadening $r_{i}=$ $r=0.5 \mathrm{~nm}$. These fits are illustrated by solid lines in Fig. 1 . The fit yields for the ML structure an average thickness of the individual isotope layers of $\sim 10.5 \mathrm{~nm}$. Standard deviations of

\begin{tabular}{|c|c|c|c|c|}
\hline Sample & $T\left({ }^{\circ} \mathrm{C}\right)$ & $t(\mathrm{~h})$ & $D_{\mathrm{Si}}\left(\mathrm{cm}^{2} \mathrm{~s}^{-1}\right)$ & Analysis \\
\hline ML & 960 & 0.5 & $2.7 \times 10^{-17}$ & NR \\
\hline ML & 940 & 0.5 & $1.1 \times 10^{-17}$ & NR \\
\hline ML & 930 & 1 & $1.3 \times 10^{-17}$ & NR \\
\hline ML & 900 & 2 & $4.6 \times 10^{-18}$ & NR \\
\hline ML & 900 & 10 & $4.1 \times 10^{-18}$ & NR \\
\hline ML & 900 & 5 & $4.2 \times 10^{-18}$ & NR \\
\hline ML & 900 & 0.5 & $3.8 \times 10^{-18}$ & NR \\
\hline ML & 870 & 6 & $1.7 \times 10^{-18}$ & NR \\
\hline ML & 850 & 15 & $8.0 \times 10^{-19}$ & NR \\
\hline ML & 830 & 30 & $2.9 \times 10^{-19}$ & NR \\
\hline ML & 815 & 72 & $1.6 \times 10^{-19}$ & NR \\
\hline ML & 800 & 120 & $1.0 \times 10^{-19}$ & NR \\
\hline ML & 800 & 300 & $1.1 \times 10^{-19}$ & NR \\
\hline ML & 800 & 50 & $1.4 \times 10^{-19}$ & NR \\
\hline$M^{a}$ & 800 & 24 & $1.0 \times 10^{-19}$ & NR \\
\hline ML & 780 & 105 & $6.8 \times 10^{-20}$ & NR \\
\hline ML & 760 & 150 & $4.6 \times 10^{-20}$ & NR \\
\hline ML & 750 & 400 & $2.4 \times 10^{-20}$ & NR \\
\hline$M^{a}$ & 750 & 168 & $2.3 \times 10^{-20}$ & NR \\
\hline ML & 742 & 501 & $2.3 \times 10^{-20}$ & NR \\
\hline$M^{a}$ & 720 & 720 & $4.9 \times 10^{-21}$ & NR \\
\hline ML & 715 & 2688 & $5.6 \times 10^{-21}$ & NR \\
\hline ML & 700 & 4320 & $1.2 \times 10^{-21}$ & NR \\
\hline $\mathrm{ML}^{\mathrm{a}}$ & 700 & 1464 & $1.2 \times 10^{-21}$ & NR \\
\hline ML & 685 & 2688 & $2.0 \times 10^{-21}$ & NR \\
\hline ML & 650 & 2853 & $5.0 \times 10^{-22}$ & NR \\
\hline
\end{tabular}
$\sigma=1.2 \mathrm{~nm}$ for structure 1 and $\sigma=2.85 \mathrm{~nm}$ for structure 2
TABLE I. Silicon self-diffusion coefficients $D_{\mathrm{Si}}$ deduced from self-diffusion experiments at the temperatures $T$ and times $t$ indicated. The diffusional broadening of the $\left({ }^{29} \mathrm{Si} /{ }^{28} \mathrm{Si}\right)_{20}$ isotope multilayer (ML) structure 1 was measured by means of neutron reflectometry (NR) with an accuracy up to $20 \%$.

${ }^{\mathrm{a}} \mathrm{ML}$ sample preannealed at $940^{\circ} \mathrm{C}$ for $0.5 \mathrm{~h}$.

were determined. The higher $\sigma$ obtained for structure 2 takes account of the higher energy of the sputter beam used for SIMS profiling of structure 2 compared to 1 (see Sec. II). Note that different settings for $r$, i.e., $r=0.1$ and $1.0 \mathrm{~nm}$, do not significantly affect $\sigma$. Solid lines in Figs. 3(a) and 3(b) illustrate best fits based on Eq. (2) to the experimental $\mathrm{Si}$ profiles obtained after diffusion annealing taking into account the respective standard deviation of the resolution function and the concentration profile $C^{*}$ given by Eqs. (3) and (4). The selfdiffusion coefficients $D_{\mathrm{Si}}$ determined from the NR and SIMS studies are listed in Tables I and II, respectively, and illustrated in Fig. 4 in comparison to literature data. Within experimental accuracy, the same self-diffusion coefficients were obtained by NR and SIMS analyses. For temperatures equal to and higher than $900{ }^{\circ} \mathrm{C}$, the data of self-diffusion deduced from structures 1 and 2 are consistent among each other and in good agreement with results on self-diffusion given in the literature. ${ }^{5}$ For temperatures below $900{ }^{\circ} \mathrm{C}$, self-diffusion in the ML structure 1 is enhanced compared to self-diffusion in SW structure 2. $D_{\mathrm{Si}}$ data obtained from structure 2 confirm the results of Shimizu et al. ${ }^{8}$ These data and the data reported by Bracht et $a l^{5}$ are considered to reflect self-diffusion under equilibrium conditions, i.e., the concentrations of $V$ and $I$ are in thermal equilibrium during annealing. In the 
TABLE II. Silicon self-diffusion coefficients $D_{\mathrm{Si}}$ deduced from self-diffusion experiments at the temperatures $T$ and times $t$ indicated. The $\left({ }^{29} \mathrm{Si} /{ }^{28} \mathrm{Si}\right)_{20}$ isotope multilayer (ML) structure 1 and the ${ }^{\text {nat }} \mathrm{Si} /{ }^{28} \mathrm{Si} /{ }^{\text {at }} \mathrm{Si}$ sandwich $(\mathrm{SW})$ structure 2 were used for the experiments. The diffusional broadening of the isotope structures was measured by means of time-of-flight secondary ion mass spectrometry (TOF-SIMS) with an accuracy of $10 \%$.

\begin{tabular}{|c|c|c|c|c|}
\hline Sample & $T\left({ }^{\circ} \mathrm{C}\right)$ & $t(\mathrm{~h})$ & $D_{\mathrm{Si}}\left(\mathrm{cm}^{2} \mathrm{~s}^{-1}\right)$ & Analysis \\
\hline $\mathrm{ML}^{\mathrm{a}}$ & 900 & 2 & $3.5 \times 10^{-18}$ & SIMS \\
\hline ML & 870 & 48 & $1.2 \times 10^{-18}$ & SIMS \\
\hline ML & 870 & 36 & $1.6 \times 10^{-18}$ & SIMS \\
\hline ML & 870 & 20 & $1.4 \times 10^{-18}$ & SIMS \\
\hline ML & 870 & 20 & $1.6 \times 10^{-18}$ & SIMS \\
\hline$M^{a}$ & 870 & 6 & $1.4 \times 10^{-18}$ & SIMS \\
\hline ML & 850 & 84 & $5.6 \times 10^{-19}$ & SIMS \\
\hline ML & 830 & 216 & $2.1 \times 10^{-19}$ & SIMS \\
\hline $\mathrm{ML}^{\mathrm{a}}$ & 830 & 30 & $2.8 \times 10^{-19}$ & SIMS \\
\hline$M^{a}$ & 815 & 72 & $1.6 \times 10^{-19}$ & SIMS \\
\hline ML & 800 & 600 & $1.1 \times 10^{-19}$ & SIMS \\
\hline ML & 800 & 201 & $1.7 \times 10^{-19}$ & SIMS \\
\hline $\mathrm{ML}^{\mathrm{a}}$ & 800 & 120 & $1.0 \times 10^{-19}$ & SIMS \\
\hline ML & 760 & 622 & $2.5 \times 10^{-20}$ & SIMS \\
\hline $\mathrm{ML}^{\mathrm{a}}$ & 750 & 168 & $1.7 \times 10^{-20}$ & SIMS \\
\hline SW & 900 & 10 & $2.8 \times 10^{-18}$ & SIMS \\
\hline SW & 870 & 120 & $7.6 \times 10^{-19}$ & SIMS \\
\hline SW & 870 & 48 & $7.9 \times 10^{-19}$ & SIMS \\
\hline SW & 850 & 120 & $4.1 \times 10^{-19}$ & SIMS \\
\hline SW & 822 & 6720 & $1.2 \times 10^{-19}$ & SIMS \\
\hline SW & 800 & 5016 & $4.7 \times 10^{-20}$ & SIMS \\
\hline SW & 785 & 6720 & $3.0 \times 10^{-20}$ & SIMS \\
\hline SW & 763 & 6552 & $1.3 \times 10^{-20}$ & SIMS \\
\hline SW & 750 & 4848 & $5.8 \times 10^{-21}$ & SIMS \\
\hline
\end{tabular}

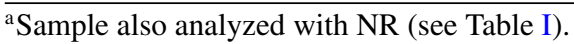

following section, a diffusion model is developed to explain the temperature dependence of self-diffusion in structures 1 and 2 that is related to self-diffusion under nonequilibrium and equilibrium conditions, respectively.

\section{A. Self-diffusion under thermal equilibrium}

\section{Analysis}

Self-diffusion data reported by Bracht et al., ${ }^{5}$ Shimizu et $a l .{ }^{8}$ and those deduced from structure 2 are considered to represent self-diffusion under thermal equilibrium. These data reveal a curved temperature dependence as illustrated in Fig. 4, accordingly, $D_{\mathrm{Si}}$ is hardly described with one single diffusion activation enthalpy. At least two different contributions with individual activation enthalpies are required to accurately reproduce the temperature dependence of $D_{\mathrm{Si}}$. A fit to the self-diffusion data ${ }^{27}$ based on the sum of two Arrhenius equations and therewith on four parameters, i.e., two diffusion prefactors and two activation enthalpies, is however not unique. Since the contribution of $I$ to self-diffusion is known from metal diffusion, first a fit with one unknown contribution to self-diffusion is performed. Taking into account the uncorrelated contribution of $I$ to self-diffusion $C_{I}^{\mathrm{eq}} D_{I} / C_{0}=$ $2980 \times \exp \left(-4.95 \mathrm{eV} / k_{B} T\right)$ derived from $\mathrm{Zn}$ diffusion ${ }^{9}$ and a correlation factor of $f_{I}=0.73$ for self-diffusion via $I,{ }^{28}$ the $I$

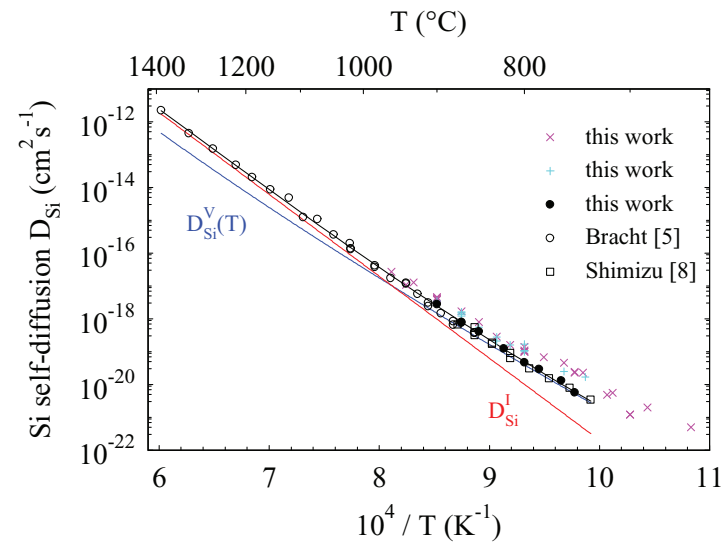

FIG. 4. (Color online) Silicon self-diffusion coefficients $D_{\mathrm{Si}}$ determined in this work $(x,+, \bullet)$ from self-diffusion experiments with $\left({ }^{29} \mathrm{Si} /{ }^{28} \mathrm{Si}\right)_{20}$ multilayers $1(x,+)$ and ${ }^{\text {nat }} \mathrm{Si} /{ }^{28} \mathrm{Si} /{ }^{\text {nat }} \mathrm{Si}$ structures $2(\bullet) .(\times)$ Results deduced from neutron reflectometry (NR) analysis of $\mathrm{Si}$ isotope multilayers (ML); (+) results from SIMS analyses of $\mathrm{Si}$ isotope multilayers; $(\bullet)$ results from SIMS analyses of ${ }^{\text {nat }} \mathrm{Si} /{ }^{28} \mathrm{Si} /{ }^{\text {nat }} \mathrm{Si}$ samples. The self-diffusion coefficients are listed in Tables I and II together with the corresponding temperatures and times. The red solid line represents the $I$ contribution $D_{\mathrm{Si}}^{I}$ to self-diffusion deduced from $\mathrm{Zn}$ diffusion in $\mathrm{Si}$ (Ref. 9). The blue solid line shows the $V$ contribution $D_{\mathrm{Si}}^{V(T)}$ to self-diffusion. The black solid is the total self-diffusion coefficient $D_{\mathrm{Si}}=D_{\mathrm{Si}}^{I}+D_{\mathrm{Si}}^{V(T)}$ given by the sum of the individual $V$ and $I$ contributions. The $V(T)$ contribution to self-diffusion comprises temperature-dependent thermodynamic properties of $V$ expressed by Eqs. (8), (10), and (11). The $V$ properties were determined from the temperature dependence of $D_{\mathrm{Si}}$ taking into account data of $D_{\mathrm{Si}}^{I}$ from metal diffusion (Ref. 9) (see Sec. III A1 for details).

contribution to self-diffusion is given by $D_{\mathrm{Si}}^{I}=f_{I} C_{I}^{\mathrm{eq}} D_{I} / C_{0}$. $C_{I}^{\mathrm{eq}}$ and $D_{I}$ are the thermal equilibrium concentration and diffusion coefficient of $I$, respectively. Taking into account this $D_{\mathrm{Si}}^{I}$ contribution, the temperature dependence of $D_{\mathrm{Si}}$ is best described by

$$
\begin{aligned}
D_{\mathrm{Si}}= & D_{\mathrm{Si}}^{I}+D_{\mathrm{Si}}^{X}=f_{I} \frac{C_{I}^{\mathrm{eq}} D_{I}}{C_{0}}+f_{X} \frac{C_{X}^{\mathrm{eq}} D_{X}}{C_{0}} \\
= & 2175.4 \times \exp \left(-\frac{4.95 \mathrm{eV}}{k_{B} T}\right) \\
& +0.0011 \times \exp \left(-\frac{3.52 \mathrm{eV}}{k_{B} T}\right) \mathrm{cm}^{2} \mathrm{~s}^{-1} .
\end{aligned}
$$

The fit is characterized by a mean-squared error of MSE $=$ $3.59 \times 10^{-2}$. Note that although most recent calculations on correlation effects in diamond structures yield $f_{I}=0.6$ for the correlation factor of self-diffusion in $\mathrm{Si},{ }^{29,30}$ we assume $f_{I}=$ 0.73 in Eq. (7) for better comparison to the results of Shimizu et al., ${ }^{8}$ who also used this value in their analysis of selfdiffusion. The first term of Eq. (7) describes the $I$-mediated self-diffusion with an activation enthalpy of $Q_{I}=4.95 \mathrm{eV}$. The second term represents a contribution of a native defect $X$ to self-diffusion with an activation enthalpy of $Q_{X}=3.52 \mathrm{eV}$. This value is in good agreement with the activation enthalpy $Q_{V}=3.6 \mathrm{eV}$ reported by Shimizu et al. and assigned to single vacancies. ${ }^{8}$ However, this activation enthalpy for $V$-mediated self-diffusion is in conflict with dopant diffusion in $\mathrm{Si}^{19}$ 


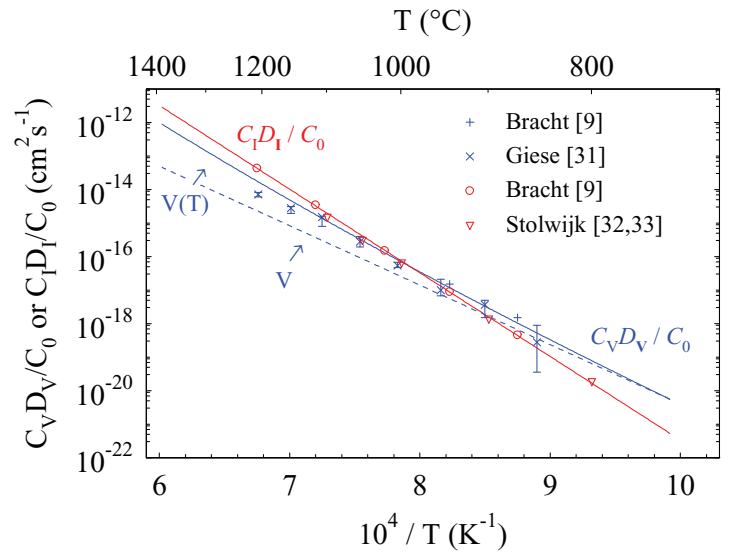

FIG. 5. (Color online) Uncorrelated individual contributions $D_{\mathrm{Si}}^{V, I} / f_{V, I}=C_{V, I}^{\mathrm{eq}} D_{V, I} / C_{0}$ of $V$ and $I$ to self-diffusion (solid and dashed lines) in comparison to the transport capacities $C_{V}^{\mathrm{eq}} D_{V} / C_{0}$ and $C_{I}^{\mathrm{eq}} D_{I} / C_{0}$ (symbols) determined from $\mathrm{Au}$ and $\mathrm{Zn}$ diffusion in Si (Refs. 9,31-33). The comparison considers diffusion correlation factors $f_{V, I}$ reported in the literature (Refs. 28, 30, and 34). The upper red solid line represents the temperature dependence of $C_{I}^{\mathrm{eq}} D_{I} / C_{0}$ obtained from $\mathrm{Zn}$ diffusion in $\mathrm{Si}$ (Ref. 9). The lower dashed blue line shows the uncorrelated $V$ contribution $D_{\mathrm{Si}}^{V} / f_{V}=C_{V}^{\mathrm{eq}} D_{V} / C_{0}$ given by the second term of Eq. (7) with $f_{V}=0.5$ (Refs. 30 and 34). This $V$ contribution clearly deviates from $C_{V}^{\mathrm{eq}} D_{V} / C_{0}$ data obtained from metal out-diffusion experiments (Ref. 31). The solid blue line describes the contribution $D_{\mathrm{Si}}^{V(T)} / f_{V}$ to self-diffusion assuming temperature-dependent thermodynamic properties of $V$. With this assumption, the temperature dependence of the experimental $C_{V}^{\mathrm{eq}} D_{V} / C_{0}$ data is better reproduced. The temperature dependence of the $I$ transport capacity is accurately described with a single diffusion activation enthalpy and expressed by $C_{I}^{\mathrm{eq}} D_{I} / C_{0}=2980 \times \exp \left(-4.95 \mathrm{eV} / k_{B} T\right)($ Ref. 9).

In addition, the assignment of $D_{\mathrm{Si}}^{X}$ to the contribution of single vacancies to self-diffusion leads to inconsistencies when the second term of Eq. (7) is compared to the uncorrelated contribution $C_{V}^{\mathrm{eq}} D_{V} / C_{0}$ of $V$ to self-diffusion determined from $\mathrm{Zn}$ out-diffusion experiments. ${ }^{31}$ Data of $C_{V}^{\mathrm{eq}} D_{V} / C_{0}$ and $C_{I}^{\mathrm{eq}} D_{I} / C_{0}$ deduced from metal-diffusion experiments are displayed in Fig. 5. The figure demonstrates that the experimental data of $C_{I}^{\mathrm{eq}} D_{I} / C_{0}$ are accurately described by the first term of Eq. (7) with $f_{I}=0.73$. However, a clear deviation is evident between $C_{V}^{\mathrm{eq}} D_{V} / C_{0}$ from metal out-diffusion (data points in Fig. 5) and $D_{\mathrm{Si}}^{X} / f_{X}=C_{X}^{\mathrm{eq}} D_{X} / C_{0}$ with $f_{X}=0.5$ (dashed line). Accordingly, the assignment of $D_{\mathrm{Si}}^{X}$ to the contribution of single vacancies to self-diffusion with the respective diffusion correlation factor $f_{V}=0.5$ (Ref. 34) is not conclusive. This is also indicated by the preexponential factor of $0.0011 \mathrm{~cm}^{2} \mathrm{~s}^{-1}$ connected with $D_{\mathrm{Si}}^{X}$. The value suggests a very low activation entropy of diffusion, which even is slightly negative. Such low values for the sum of the migration and formation entropy of $V$ in $\mathrm{Si}$ are rather unlikely and even at variance with the thermodynamics of point defects in solids. More realistic values for the diffusion activation entropy are obtained assuming three different contributions to self-diffusion. Again, we consider the $I$ contribution to self-diffusion from the metal diffusion. The fit is characterized by $\mathrm{MSE}=4.44 \times 10^{-2}$ and diffusion activation enthalpies (entropies) of $4.77 \mathrm{eV}\left(8.1 k_{B}\right)$ and $3.78 \mathrm{eV}\left(2.3 k_{B}\right)$. An assignment of these contributions to, e.g., monovacancies and divacancies, is also not conclusive because the diffusion activation enthalpy deduced for the divacancy is too small compared to realistic estimates. ${ }^{35-37}$ The formation energy of the divacancy in $\mathrm{Si}$ is theoretically calculated to be above $5 \mathrm{eV}$. Together with a migration energy of the divacancy in the range of $1 \mathrm{eV},{ }^{37}$ an activation enthalpy of $6-7 \mathrm{eV}$ is to be expected. Hence, the approaches assuming two or three contributions to self-diffusion do not provide a convincing interpretation of the temperature dependence of self-diffusion.

The concept considered so far to describe the temperature dependence of self-diffusion assumes temperatureindependent thermodynamic properties of $I$ and $V$. This seems to be justified for $I$ since the uncorrelated contribution of $I$ to self-diffusion determined from metal diffusion is accurately described by a single activation enthalpy even for temperatures down to $800{ }^{\circ} \mathrm{C}$ (see Fig. 5). The $V$ contribution to self-diffusion $D_{\mathrm{Si}}^{V}$ is less accurately determined because the $V$ fraction in experiments on in-diffusion of $\mathrm{Au}$ and $\mathrm{Zn}$ in $\mathrm{Si}$ is minor compared to the fraction of $I .{ }^{9,38}$ In the case of metal outdiffusion, where mainly $V$ mediate the out-diffusion process, the formation of microscopic defects impede an accurate determination of $D_{\mathrm{Si}}^{V} \cdot{ }^{31}$ Accordingly, considering the lack of data for $D_{\mathrm{Si}}^{V}$, a temperature dependence of the thermodynamic properties of $V$ can not be excluded. Following an earlier interpretation of Si self-diffusion suggested by Seeger et al., ${ }^{39}$ a linear dependence of the $V$-formation enthalpy $H_{V}^{F}$ and $V$-migration enthalpy $H_{V}^{M}$ on temperature is assumed:

$$
H_{V}^{F, M}(T)=H_{V}^{F, M}\left(T_{0}\right)+\alpha_{V}^{F, M}\left(T-T_{0}\right) .
$$

The enthalpy $H_{V}^{F, M}\left(T_{0}\right)$ and the temperature coefficient $\alpha_{V}^{F, M}$ are independent of temperature. $T_{0}$ denotes a reference temperature. For constant pressure conditions, the thermodynamic relation

$$
\left(\frac{\partial S_{V}^{F, M}}{\partial T}\right)_{p}=\frac{1}{T}\left(\frac{\partial H_{V}^{F, M}}{\partial T}\right)_{p}
$$

is fulfilled, yielding the following temperature dependence of the $V$-formation entropy $S_{V}^{F}$ and $V$-migration entropy $S_{V}^{M}$ :

$$
S_{V}^{F, M}(T)=S_{V}^{F, M}\left(T_{0}\right)+\alpha_{V}^{F, M} \ln \left(T / T_{0}\right) .
$$

With Eqs. (8) and (10), the $V$ contribution to self-diffusion is given by

$$
\begin{aligned}
D_{\mathrm{Si}}^{V}= & f_{V} g_{V} a_{0}^{2} v_{V 0} \exp \left(\frac{S_{V}^{F}(T)+S_{V}^{M}(T)}{k_{B}}\right) \\
& \times \exp \left(-\frac{H_{V}^{F}(T)+H_{V}^{M}(T)}{k_{B} T}\right)
\end{aligned}
$$

with the diffusion correlation factor $f_{V}=0.5$ and geometry factor $g_{V}=\frac{1}{8}$ for the diamond structure ${ }^{34}$ and the silicon lattice constant $a_{0}=0.5431 \mathrm{~nm}$. The attempt frequency $v_{V 0}$ can be calculated assuming a periodic lattice potential that is harmonic at the Si-lattice sites by means of ${ }^{40}$

$$
v_{V 0}=\frac{2}{a_{0}}\left(\frac{2 H_{V}^{M}(T)}{3 m_{\mathrm{Si}}}\right)^{0.5} .
$$


$m_{\mathrm{Si}}$ represents the mass of the $\mathrm{Si}$ atom. The temperature dependence of $H_{V}^{M}$ given by Eq. (8) is considered in Eq. (12) for the calculation of the attempt frequency.

The upper black solid line in Fig. 4 shows the calculated temperature dependence of self-diffusion $D_{\mathrm{Si}}=D_{\mathrm{Si}}^{I}+$ $D_{\mathrm{Si}}^{V}$ assuming $D_{\mathrm{Si}}^{I}$ from metal diffusion and a $D_{\mathrm{Si}}^{V}$ contribution with temperature-dependent thermodynamic properties of $V . D_{\mathrm{Si}}^{I}=f_{I} C_{I}^{\mathrm{eq}} D_{I} / C_{0}$ is given by $C_{I}^{\mathrm{eq}} D_{I} / C_{0}=2980 \times$ $\exp \left(-4.95 \mathrm{eV} / k_{B} T\right) \mathrm{cm}^{2} \mathrm{~s}^{-1}$ and the correlation factor $f_{I}=$ 0.60 that recently was determined by Posselt et al. ${ }^{29,30}$ for $I$-mediated self-diffusion. This contribution is illustrated by the red solid line in Fig. 4. The $V$ contribution was determined by fitting the sum of $D_{\mathrm{Si}}^{I}$ and $D_{\mathrm{Si}}^{V}$ to $D_{\mathrm{Si}}$. A reference temperature of $T_{0}=700{ }^{\circ} \mathrm{C}$ and suitable settings for the formation and migration enthalpy (entropy) of $H_{V}^{F}\left(T_{0}\right)=3.16 \mathrm{eV}$ and $H_{V}^{M}\left(T_{0}\right)=0.5 \mathrm{eV}\left[S_{V}^{F}\left(T_{0}\right)=1 k_{B}\right.$ and $\left.S_{V}^{M}\left(T_{0}\right)=1 k_{B}\right]$ were assumed to meet the properties of single isolated $V$ at low temperatures. ${ }^{17,18}$ The temperature coefficients $\alpha_{V}^{F}$ and $\alpha_{V}^{M}$ were varied to reproduce the temperature dependence of $D_{\mathrm{Si}}$. The $V$ contribution, i.e., $D_{\mathrm{Si}}^{V}$ illustrated by the blue solid line in Fig. 4 is characterized by $\alpha_{V}^{F}=1.0 \times 10^{-3} \mathrm{eV} / \mathrm{K}$ and $\alpha_{V}^{M}=5.0 \times 10^{-4} \mathrm{eV} / \mathrm{K}$. With these settings, the temperature dependence of $D_{\mathrm{Si}}$ is accurately described as illustrated by the black solid line in Fig. 4. The line is characterized by a $\mathrm{MSE}=5.6 \times 10^{-2}$.

The description of self-diffusion with temperaturedependent thermodynamic properties of $V$ provides a good agreement to the uncorrelated $V$ contribution to self-diffusion deduced from metal out-diffusion studies as demonstrated in Fig. 5 by the blue solid line marked with $V(T)$ and the experimental data of $C_{V}^{\mathrm{eq}} D_{V} / C_{0}$. The formation $H_{V}^{F}\left(S_{V}^{F}\right)$ and migration $H_{V}^{M}\left(S_{V}^{M}\right)$ enthalpy (entropy) of $V$ as well as the diffusion activation enthalpy $Q_{V}=H_{V}^{F}+H_{V}^{M}$ (entropy $S_{V}=S_{V}^{F}+S_{V}^{M}$ ) of self-diffusion are illustrated in Fig. 6 as function of temperature with $T \geqslant T_{0}=700^{\circ} \mathrm{C}$. The formation (migration) enthalpy of $V$ increases from $3.16 \mathrm{eV}(0.5 \mathrm{eV})$ to $3.86 \mathrm{eV}(0.85 \mathrm{eV})$ for temperatures between $700{ }^{\circ} \mathrm{C}$ and $1400^{\circ} \mathrm{C}$. In the same temperature range, the activation enthalpy $Q_{V}$ of self-diffusion via $V$ increases from 3.66 to $4.71 \mathrm{eV}$. On the other hand, the formation (migration) entropy of $V$ increases from $1 k_{B}\left(1 k_{B}\right)$ to $7.3 k_{B}\left(4.1 k_{B}\right)$, leading to an

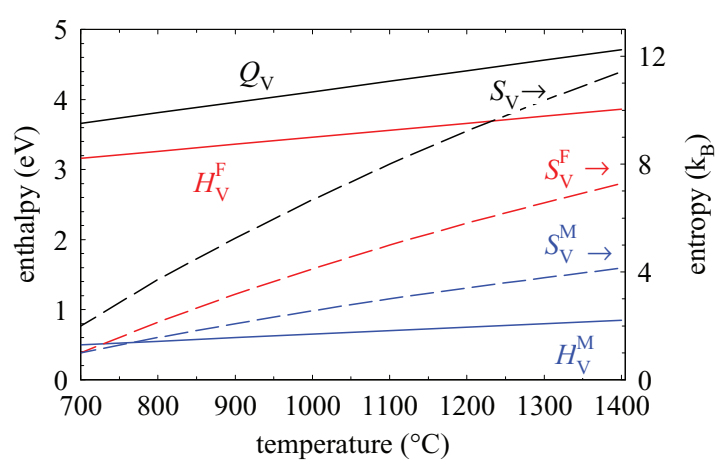

FIG. 6. (Color online) Temperature dependence of the thermodynamic properties of $V$ in Si deduced from the temperature dependence of $\mathrm{Si}$ self-diffusion. The activation enthalpy (entropy) $Q_{V}\left(S_{V}\right)$ of self-diffusion via $V$, which is given by the sum of the enthalpy (entropy) of $V$ formation $H_{V}^{F}\left(S_{V}^{F}\right)$ and migration $H_{V}^{M}\left(S_{V}^{M}\right)$, is referred to the left (right) $y$ axis. increase of the activation entropy $S_{V}$ from $2 k_{B}$ to $11.4 k_{B}$. The significance of these temperature-dependent thermodynamic properties of $V$ is discussed in the next section.

\section{Discussion}

The self-diffusion in the ${ }^{\text {nat }} \mathrm{Si} /{ }^{28} \mathrm{Si} /{ }^{\text {nat }} \mathrm{Si} \mathrm{SW}$ structure 2 fully verifies the data reported by Shimizu et al ${ }^{8}$ for self-diffusion at temperatures below $900{ }^{\circ} \mathrm{C}$. The excellent agreement between both sets of data obtained from two independent studies led us to conclude that these results are representative for thermal equilibrium conditions. In order to describe the temperature dependence of self-diffusion, two and three different native defects were considered to mediate the diffusion process (see Sec. III A1). An attempt to describe the temperature dependence of self-diffusion on the basis of a physical model that assumes a single activation enthalpy for each individual contribution to self-diffusion failed as the deduced activation energies are too low to represent self-diffusion via monovacancies and/or divacancies (see Sec. III A1). The insufficiency of this approach also shows up in an exceptional low preexponential factor of the contribution mediating selfdiffusion at low temperatures. This factor reveals a too low or even negative activation entropy $S_{X}$ for self-diffusion via the defect $X$. An assignment of $X$ to $V$ bears a conflict with $V$-mediated dopant diffusion in Si. For example, the diffusion of antimony ( $\mathrm{Sb}$ ) in $\mathrm{Si}$ is mainly mediated by $V$ (Ref. 41) and described by an activation enthalpy of $4.08 \mathrm{eV}{ }^{42}$ Assuming a difference between the activation enthalpy of self- and dopant diffusion in $\mathrm{Si}$ to be similar to that of $\mathrm{Ge},{ }^{43}$ that is, 0.3 to $0.6 \mathrm{eV}^{44}$ the activation enthalpy of $V$-mediated self-diffusion in $\mathrm{Si}$ should be in the range of 4.4 to $4.7 \mathrm{eV}$ (Ref. 19) rather than below $4 \mathrm{eV}$. The temperature dependence of self-diffusion illustrated in Fig. 4 is hardly described with $Q_{V}>4 \mathrm{eV}$. This led to the legitimate question on the actual contribution of $V$ to self-diffusion. The present approach to unravel this mystery of self-diffusion considers temperature-dependent thermodynamic properties of $V$, that is, the formation and migration enthalpy of $V$ change with temperature. Compared to $V$, the thermodynamic properties of $I$ are assumed to be independent of temperature. This is supported by the $I$ contribution to self-diffusion that is accurately described with a single diffusion activation enthalpy (see Fig. 5). Based on these assumptions for $V$ and $I$, the temperature dependence of the $V$-related thermodynamic properties is deduced from the equilibrium self-diffusion data illustrated in Fig. 4. Fitting of the experimental results (see Sec. III A1 for details) yields the temperature dependence of the $V$ properties displayed in Fig. 6. The activation enthalpy (entropy) $Q_{V}\left(S_{V}\right)$ of $V$-mediated self-diffusion varies from $3.7 \mathrm{eV}\left(2.0 k_{B}\right)$ at $700{ }^{\circ} \mathrm{C}$ to $4.7 \mathrm{eV}\left(11.4 k_{B}\right)$ at $1400{ }^{\circ} \mathrm{C}$. In particular, for temperatures above $900{ }^{\circ} \mathrm{C}$, where most dopant diffusion studies have been performed, the temperature dependence of $Q_{V}$ suggests values of $>4 \mathrm{eV}$ that are in good agreement with $V$-mediated dopant diffusion in $\mathrm{Si}$.

The values given for $Q_{V}$ and $S_{V}$ in Fig. 6 describe a vacancy, whose structure is localized at low and more extended at high temperatures. Such a spread-out or strongly relaxed point defect was first proposed by Seeger and $\mathrm{Chik}^{40}$ to explain the high activation entropy of self-diffusion in $\mathrm{Si}$ and Ge. 
However, the concept of spread-out point defects could not be verified due to experimental difficulties that have limited investigations of self-diffusion to a rather narrow temperature range. Recently, Hüger et al. ${ }^{45}$ have extended self-diffusion experiments in Ge to temperatures as low as $429{ }^{\circ} \mathrm{C}$ by means of NR measurements. Interestingly, self-diffusion in $\mathrm{Ge}$ is accurately described over nine orders of magnitude with a single diffusion activation enthalpy of $3.13 \mathrm{eV}^{45}$ This indicates that in the case of $\mathrm{Ge}$, one single mechanism dominates self-diffusion and that the thermodynamic properties of the corresponding native defect does not significantly change with temperature. Additional information on the impact of hydrostatic pressure and doping on self-diffusion ${ }^{46}$ as well as on the interference between self- and dopant diffusion ${ }^{47,48}$ reveals that $V$ mediate self-diffusion in Ge. This interpretation is also supported by atomistic calculations. ${ }^{49,50}$ Back to the case of $\mathrm{Si}$, the experimental data on self-diffusion also span nine orders of magnitude and clearly reveal the contribution of at least two native defects to self-diffusion. The interpretation of the temperature dependence of self-diffusion with two individual contributions, whose thermodynamic properties are constant with temperature, leads to inconsistencies to dopant diffusion. These inconsistencies are solved when the thermodynamic properties of at least one native defect changes with temperature. It is assumed that the $V$ properties change with temperature because present experimental data on the I contribution to self-diffusion do not justify a temperature dependence of the $I$ properties (see Fig. 5), although, such a dependence is possible since different interstitial configurations have been identified theoretically (see, e.g., Ref. 51), whose dominance may change with temperature. Whether the thermodynamic properties of only one native point defect (either $V$ or $I$ ) or of both native defects depend on temperature can not be solved definitely by means of the self-diffusion data available until now. However, the concept of temperaturedependent $V$ properties could be verified by studying the diffusion of, e.g., $\mathrm{Sb}$ in $\mathrm{Si}$, that is known to be mainly mediated by $V .41,43,52-54$ However, in contrast to previous studies, the diffusion of $\mathrm{Sb}$ should be investigated for temperatures between $700{ }^{\circ} \mathrm{C}$ and $1400{ }^{\circ} \mathrm{C}$ to identify possible deviations of $\mathrm{Sb}$ diffusion from a single Arrhenius-type temperature dependence. This study should represent thermal equilibrium and electronic intrinsic conditions to avoid any impact on diffusion due to nonequilibrium concentrations of native point defects and Fermi-level effects.

Finally, we would like to comment on the concept of effective diffusion energies proposed by Pochet and Caliste ${ }^{37,55}$ to solve the discrepancies found in the literature on the $V$ migration energy at low and high temperatures. Their numerical studies of self-diffusion via monovacancies and divacancies combine structure calculations by ab initio and Monte Carlo methods. The calculations demonstrate that the effective diffusion behavior of $V$ via transient formation of divacancies can be non-Arrhenian depending on the temperature range and the $V$ concentration. Based on this concept, the authors provide a plausible interpretation of the high-migration enthalpy of $(1.8 \pm 0.5) \mathrm{eV}$ deduced from self-diffusion experiments under proton irradiation, ${ }^{56}$ that is, the migration enthalpy assigned to a monovacancy rather reflects an effective migration energy due to the transient formation of divacancies. The formation of divacancies is plausible since proton irradiation realizes $V$ concentrations in Si that exceed those under thermal equilibrium by several orders of magnitude. However, the concept of effective diffusion suggested by Caliste and Pochet ${ }^{55}$ is not applicable for Si self-diffusion under thermal equilibrium. Experimental estimates of the $V$ concentration in $\mathrm{Si}$ yield concentrations in the range of $10^{15} \mathrm{~cm}^{-3}$ at the melting point. Metal-diffusion experiments provide an upper bound of about $2 \times 10^{14} \mathrm{~cm}^{-3}$ for the $V$ concentration at $870{ }^{\circ} \mathrm{C}$ (Ref. 9) with even lower values for lower temperatures. The calculations of Pochet and Caliste et al. ${ }^{37,55}$ assume $V$ concentrations of $10^{16} \mathrm{~cm}^{-3}$ that clearly exceed the thermal equilibrium concentration of $V$. Considering two orders of magnitude lower concentrations, the intermediate diffusion regime, where diffusion of monovacancies is affected by the transient formation of divacancies, will shift to temperatures beyond those accessible by equilibrium self-diffusion studies. ${ }^{55}$ Accordingly, an effective self-diffusion via monovacancies and divacancies under thermal equilibrium is not conclusive.

\section{B. Self-diffusion under nonequilibrium conditions}

\section{Analysis}

NR and SIMS analyses of self-diffusion in the ML structure 1 clearly reveal an enhanced diffusion compared to selfdiffusion in the SW structure 2. This, in particular, holds for temperatures below $900{ }^{\circ} \mathrm{C}$. An impact of doping on self-diffusion, that is generally known to affect self-diffusion in semiconductors (see, e.g., Ref. 11), can be excluded as the isotope ML structure is undoped. Although the Si substrate wafer is doped with $\mathrm{B}$ to $2 \times 10^{15} \mathrm{~cm}^{-3}$ and $\mathrm{B}$ can diffuse into the isotope ML during annealing, the B-doping level is too low to significantly affect the position of the Fermi level and therewith the concentration of charged native defects. Accordingly, doping effects are excluded as an explanation of the enhanced self-diffusion.

Self-diffusion experiments performed at the same temperature for various times and a preanneal of the ML structure at $940{ }^{\circ} \mathrm{C}$ for $30 \mathrm{~min}$ did not significantly change self-diffusion, i.e., the enhanced self-diffusion is conserved. Since both structures No. 1 and No. 2 were annealed under identical experimental conditions (see Sec. II), the enhanced selfdiffusion in structure 1 can not be related to environmental conditions. Accordingly, the differences observed in the behavior of self-diffusion in structures 1 and 2 grown by MBE and CVD, respectively, must be due to differences in the microstructure. Although we could not detect any significant structural differences between these samples by means of transmission electron microscopy (TEM), small defect clusters invisible by TEM and consisting of either $I$ or $V$ aggregates likely cause the enhanced self-diffusion. Such defect clusters could have evolved with the incorporation of carbon during the MBE growth of structure No. 1 since this sample exhibits a high carbon concentration of $3 \times 10^{18} \mathrm{~cm}^{-3}$ (see Sec. II). Carbon in $\mathrm{Si}$ is known to act as trap for $I$ (Refs. 57 and 58) and thus can favor the formation of $I$-related clusters. On the other hand, $V$-related defect clusters can evolve during the MBE growth initiated by the attractive $V-V$ pairing. ${ }^{36,55}$ Theoretical calculations on the formation and binding energies of small $V$ - and $I$-related defect clusters reveal their structure 
and stability. ${ }^{36,59}$ Since self-diffusion is directly mediated by native point defects, the enhanced self-diffusion in structure 1 provides direct evidence of defect clusters that inject native defects during annealing. This is supported in the following, more quantitatively, by numerical simulations of self-diffusion under the impact of the dissolution of $V$ - and $I$-related clusters. The mathematical formulation is based on the following differential equations:

$$
\begin{gathered}
\frac{\partial C_{V}}{\partial t}-D_{V} \frac{\partial^{2} C_{V}}{\partial x^{2}}=k_{0}^{V}-k_{+} C_{V} C_{I}+k_{-} C_{0} C_{0}, \\
\frac{\partial C_{I}}{\partial t}-D_{I} \frac{\partial^{2} C_{I}}{\partial x^{2}}=k_{0}^{I}-k_{+} C_{V} C_{I}+k_{-} C_{0} C_{0}, \\
\frac{\partial C_{\mathrm{Si}}}{\partial t}-\frac{\partial}{\partial x} D_{\mathrm{Si}} \frac{\partial C_{\mathrm{Si}}}{\partial x}=0 .
\end{gathered}
$$

$C_{V}$ and $C_{I}$ are the local $V$ and $I$ concentrations and $C_{0}=$ $5 \times 10^{22}$ the Si atom density. $D_{V}$ and $D_{I}$ are the diffusion coefficients of single $V$ and $I$, respectively. The parameters $k_{+}$and $k_{-}$denote the rate constants for the Frenkel pair reaction $V+I \leftrightharpoons\{0\}$ in the forward and backward directions, respectively. Applying the law of mass action to the Frenkel pair reaction, the rate constants $k_{+}$and $k_{-}$are interrelated via

$$
\frac{k_{+}}{k_{-}}=\frac{C_{0} C_{0}}{C_{V}^{\mathrm{eq}} C_{I}^{\mathrm{eq}}} .
$$

This equation allows us to replace $k_{-}$in Eqs. (13) and (14) by $k_{+} C_{V}^{\mathrm{eq}} C_{I}^{\mathrm{eq}} /\left(C_{0} C_{0}\right)$. Assuming a diffusion-limited annihilation of $V$ and $I, k_{+}$is given by $4 \pi r\left(D_{V}+D_{I}\right),{ }^{60}$ where $r$ represents the capture radius. The capture radius is of the dimension of the Si lattice constant $\left(a_{0}=5.431 \AA\right)$ and set to $r=1.0 \times a_{0} . k_{0}^{V}$ and $k_{0}^{I}$ in Eqs. (13) and (14) are the production rates of $V$ and $I$, respectively, due to the dissolution of the defect clusters. These production rates depend on temperature because the stability of defect clusters decreases with increasing temperature. Equation (15) describes the self-diffusion with the local self-diffusion coefficient given by

$$
\begin{aligned}
D_{\mathrm{Si}} & =\left(f_{V} C_{V} D_{V}+f_{I} C_{I} D_{I}\right) / C_{0} \\
& =\left(f_{V} C_{V}^{\mathrm{eq}} D_{V} \xi_{V}+f_{I} C_{I}^{\mathrm{eq}} D_{I} \xi_{I}\right) / C_{0} \\
& =D_{\mathrm{Si}}^{V} \xi_{V}+D_{\mathrm{Si}}^{I} \xi_{I},
\end{aligned}
$$

where $\xi_{V, I}=C_{V, I}(x, t) / C_{V, I}^{\mathrm{eq}}$ represents the local concentration of $V$ and $I$ normalized to the thermal equilibrium concentration $C_{V, I}^{\mathrm{eq}}$. The diffusion correlation factors for selfdiffusion via $V$ and $I$ are set to $f_{V}=0.5$ and $f_{I}=0.60$ according to recent calculations on correlation effects in diamond structures. ${ }^{29,30}$ The contribution of $D_{\mathrm{Si}}^{V}$ to self-diffusion is calculated by means of Eqs. (8), (10), and (11) taking into account the parameter settings from self-diffusion under thermal equilibrium. The $I$ contribution to self-diffusion is given by $D_{\mathrm{Si}}^{I}=f_{I} C_{I}^{\mathrm{eq}} D_{I} / C_{0}=1788 \times \exp \left(-4.95 \mathrm{eV} / k_{B} T\right) \mathrm{cm}^{2} \mathrm{~s}^{-1}$ with $C_{I}^{\mathrm{eq}} D_{I} / C_{0}$ from metal diffusion ${ }^{9}$ and $f_{I}=0.6 .^{30}$ The temperature dependencies of $C_{I}^{\mathrm{eq}}$ and $C_{V}^{\mathrm{eq}}$ were calculated via $C_{I}^{\mathrm{eq}}=2.9 \times 10^{24} \times \exp \left(-3.18 \mathrm{eV} / k_{B} T\right) \mathrm{cm}^{-3}$ (Ref. 9) and $C_{V}^{\mathrm{eq}}=C_{0} \times \exp \left[S_{V}^{F}(T) / k_{B}\right] \times \exp \left[-H_{V}^{F}(T) / k_{B} T\right]$, respectively, taking into account Eqs. (8) and (10) for $H_{V}^{F}(T)$ and $S_{V}^{F}(T)$ whose temperature dependence is illustrated in Fig. 6. Equations (13) to (15) were solved numerically. Concentration profiles of Si were calculated with $k_{0, V}$ and $k_{0, I}$ as adjustable parameters to describe the experimental profiles of structure 1. As initial condition, the concentrations of $V$ and $I$ are considered to be in thermal equilibrium, i.e., $C_{V, I}(x, t=0)=C_{V, I}^{\mathrm{eq}}$. The $\mathrm{Si}$ surface is assumed to be an effective sink/source for vacancies, i.e., $C_{V}(x=0, t)=C_{V}^{\mathrm{eq}}$, whereas a limited efficiency for the annihilation of $I$ at the surface is taken into account:

$$
\left.\frac{C_{I}^{\mathrm{eq}} D_{I}}{C_{0}} \frac{\partial C_{I}}{\partial x}\right|_{x=0}=v_{I}\left(C_{I}-C_{I}^{\mathrm{eq}}\right)
$$

with $v_{I}=5 \times 10^{-4} \mathrm{~cm} / \mathrm{s}$. A setting of $v_{I}=0$ represents fully reflecting boundary conditions. A limited efficiency for defect annihilation at the surface must be assumed to explain the almost homogeneously enhanced self-diffusion observed in structure 1. This depth-independent behavior of self-diffusion demonstrates nearly constant concentrations of $I$ and $V$. A similar behavior of $I$ was postulated by Gossmann et al ${ }^{61}$ to explain the differences in dopant diffusion of B- and Sb-doped $\mathrm{Si}$ superlattices annealed in vacuum and argon at $810^{\circ} \mathrm{C}$. A limited efficiency of a free surface to annihilate $I$ formed in the bulk by proton radiation was also verified in the case of Ge. ${ }^{62,63}$ Electronic states at the Ge surface were proposed to control the $I$ surface annihilation. ${ }^{63}$ A similar behavior in the annihilation of $I$ becomes evident by the enhanced self-diffusion in structure 1, although, recent investigations of self-diffusion in Si under proton irradiation did not reveal any limited defect annihilation at the surface. ${ }^{56}$ The reason for the differences in the efficiency of defect annihilation at the $\mathrm{Si}$ surface remains unsolved. Additional diffusion studies under various boundary conditions are required to solve this issue. This is, however, beyond the scope of this paper.

Figure 7 shows a $\mathrm{Si}$ self-diffusion profile measured with SIMS after annealing of structure 1 at $815{ }^{\circ} \mathrm{C}$ for $72 \mathrm{~h}$. Solid lines illustrate numerical solutions of Eqs. (13) to (15) assuming the above-mentioned initial and boundary conditions. The experimental profile is accurately described by the simulation. The corresponding concentration profiles of $V$ and $I$ normalized to the respective equilibrium concentrations are indicated by the blue and red lines, respectively. The

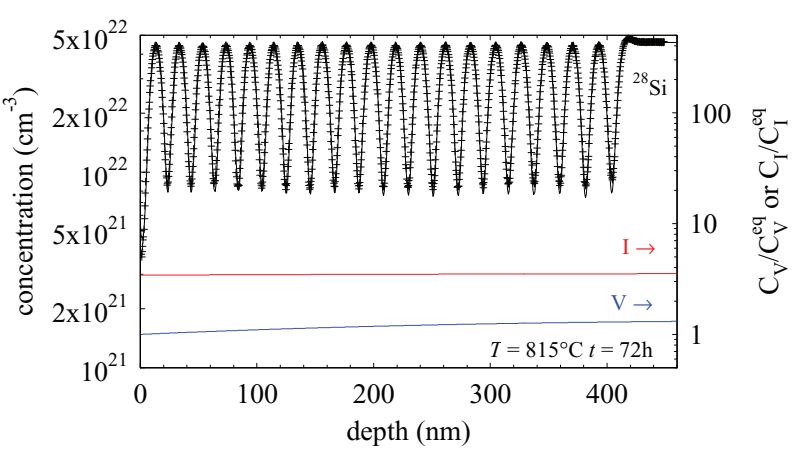

FIG. 7. (Color online) SIMS concentration profile $(+)$ of ${ }^{28} \mathrm{Si}$ measured after annealing of the $\left({ }^{29} \mathrm{Si} /{ }^{28} \mathrm{Si}\right)_{20}$ isotope multilayer at the temperature and time indicated. The black solid line shows a successful simulation of the enhanced self-diffusion in the ML structure that is based on the data of Si self-diffusion for equilibrium conditions and assumes the dissolution of $V$ - and $I$-related defect clusters. The corresponding local supersaturations $S_{V, I}=C_{V, I} / C_{V, I}^{\mathrm{eq}}$ of $V$ (blue line) and $I$ (red line) calculated by solving Eqs. (13) to (15) numerically are given by the right $y$ axis. 
TABLE III. Rate constants $k_{0}^{V}$ and $k_{0}^{I}$ for the formation of $V$ and $I$ in $\mathrm{Si}$, respectively, deduced from modeling $\mathrm{Si}$ self-diffusion in $\left({ }^{29} \mathrm{Si} /{ }^{28} \mathrm{Si}\right)_{20}$ isotope multilayers (see Fig. 7). The Si profiles were measured with SIMS after annealing at the temperatures $T$ and times $t$ indicated. The self-diffusion model considers the dissolution of $V$ and $I$-defect clusters. The binding enthalpy of the defect cluster is given by the temperature dependence of the respective rate constant (see Fig. 8).

\begin{tabular}{lrcc}
\hline \hline$T\left({ }^{\circ} \mathrm{C}\right)$ & $t(\mathrm{~min})$ & $k_{0}^{V}\left(\mathrm{~s}^{-1}\right)$ & $k_{0}^{I}\left(\mathrm{~s}^{-1}\right)$ \\
\hline 900 & 2 & $(2.0 \pm 0.2) \times 10^{-8}$ & $(8.0 \pm 0.4) \times 10^{-10}$ \\
870 & 20 & $(3.0 \pm 0.3) \times 10^{-9}$ & $(5.1 \pm 0.5) \times 10^{-10}$ \\
870 & 20 & $(4.0 \pm 0.2) \times 10^{-9}$ & $(5.7 \pm 0.1) \times 10^{-10}$ \\
870 & 48 & $(4.9 \pm 0.2) \times 10^{-9}$ & $(3.1 \pm 0.1) \times 10^{-10}$ \\
870 & 6 & $(2.8 \pm 0.4) \times 10^{-9}$ & $(5.6 \pm 0.3) \times 10^{-10}$ \\
870 & 36 & $(5.4 \pm 1.1) \times 10^{-9}$ & $(3.4 \pm 0.1) \times 10^{-10}$ \\
850 & 84 & $(3.5 \pm 0.4) \times 10^{-9}$ & $(3.0 \pm 0.1) \times 10^{-10}$ \\
830 & 216 & $(1.0 \pm 0.3) \times 10^{-9}$ & $(9.2 \pm 0.3) \times 10^{-11}$ \\
830 & 30 & $(2.9 \pm 0.7) \times 10^{-9}$ & $(1.8 \pm 0.4) \times 10^{-10}$ \\
815 & 72 & $(1.3 \pm 0.7) \times 10^{-9}$ & $(1.4 \pm 0.1) \times 10^{-10}$ \\
800 & 600 & $(4.5 \pm 0.1) \times 10^{-10}$ & $(1.4 \pm 0.1) \times 10^{-10}$ \\
800 & 201 & $(7.5 \pm 0.9) \times 10^{-10}$ & $(2.5 \pm 0.2) \times 10^{-10}$ \\
800 & 120 & $(5.1 \pm 0.2) \times 10^{-10}$ & $(1.3 \pm 0.1) \times 10^{-10}$ \\
760 & 622 & $(1.5 \pm 0.4) \times 10^{-10}$ & $(5.2 \pm 0.3) \times 10^{-11}$ \\
740 & 168 & $(7.3 \pm 0.4) \times 10^{-11}$ & $(1.8 \pm 0.2) \times 10^{-11}$ \\
\hline \hline
\end{tabular}

dissolution of $V$ and $I$ clusters gives rise to local concentrations that deviate from thermal equilibrium, i.e., $\xi_{V, I} \neq 1$. The concentration of $V$ at $x=0$ equals $C_{V}^{\mathrm{eq}}$, i.e., $\xi_{V}=1$, in accord with the assumed boundary condition whereas the boundary concentration of $I$ slightly deviates from equilibrium, i.e., $\xi_{I}>1$. The local concentrations established for $V$ and $I$ lead to the enhanced self-diffusion [see Eq. (17)]. The rate constants $k_{0}^{V}$ and $k_{0}^{I}$ deduced from modeling Si self-diffusion in structure 1 are listed in Table III and illustrated in Fig. 8 as function

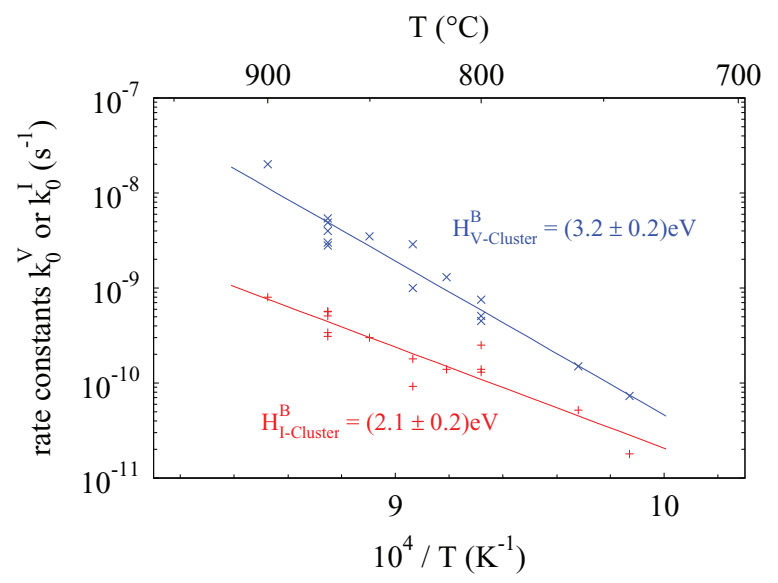

FIG. 8. (Color online) Rate constants $k_{0}^{I}(\mathrm{red}+)$ and $k_{0}^{V}$ (blue $\times$ ) of $I$ - and $V$-related defect clusters as function of the inverse temperature. The rate constants were deduced from modeling the enhanced self-diffusion in $\left({ }^{29} \mathrm{Si} /{ }^{28} \mathrm{Si}\right)_{20}$ isotope multilayers. The enhanced self-diffusion is considered to be caused by the dissolution of $I$ - and $V$-related defect clusters. The temperature dependence of $k_{0}^{I}$ and $k_{0}^{V}$ reveals the binding energy $H_{I \text { cluster }}^{B}$ and $H_{V \text { cluster }}^{B}$ of $I$ and $V$ clusters, respectively, indicated in the figure. of the inverse temperature. The temperature dependencies of $k_{0}^{V}$ and $k_{0}^{I}$ are described with Arrhenius expressions yielding activation enthalpies of $(3.2 \pm 0.2) \mathrm{eV}$ and $(2.1 \pm 0.2) \mathrm{eV}$ for the dissolution of the $V$ and $I$ clusters, respectively. These enthalpies reflect the binding energy $H_{V, I}^{B}$ clusters of the defect cluster. These binding energies are compared in the next section with results given in literature.

\section{Discussion}

The enhanced self-diffusion in the isotope multilayer structure 1 provides direct evidence of native defect clusters that dissolve during annealing. Thereby, a supersaturation of native defects is established that leads to enhanced self-diffusion. Numerical simulation yields binding enthalpies of $(3.2 \pm 0.2) \mathrm{eV}$ and $(2.1 \pm 0.2) \mathrm{eV}$ for the $V$ and $I$ clusters, respectively. Obviously, the $V$ clusters are more stable than the $I$ clusters. This result is in accurate agreement with the stability of $V$ - and $I$-related defect clusters that was deduced from the analysis of transient enhanced diffusion of dopants in Si (see, e.g., Ref. 51 and references therein). Pelaz et al. ${ }^{51}$ reviewed experimental and theoretical results of the thermodynamic properties of $V$ and $I$ clusters in Si. Generally, it is observed that small defect clusters exhibit higher stabilities for specific cluster sizes, whereas the stability of larger clusters seems to level off. ${ }^{51}$ In particular, theory predicts values around $3 \mathrm{eV}$ for the binding energy of $V$ clusters with 6,8-10, and more than 15 vacancies. ${ }^{36}$ The binding energy of large $V$ clusters is verified by experimental results of Kalyanaraman et al. ${ }^{64}$ and Venezia et al. ${ }^{65}$ For example, Kalyanaraman et al. ${ }^{64}$ studied the evaporation of $V$ from clusters formed by $\mathrm{Si}$ implantation with a $\mathrm{Au}$ labeling technique and determined a binding energy of $V$ to clusters of $(3.2 \pm 0.2) \mathrm{eV}$. The $T$ dependence of $k_{0}^{V}$ (see previous section) exhibits the same binding enthalpy. On the other hand, the stability of $I$ clusters derived from the $T$ dependence of $k_{0}^{I}$ also confirms results given in the literature. ${ }^{51}$ This consistency obtained between this work and the literature on the binding energies for $V$ and $I$ clusters supports the proposed defect model that describes the enhanced self-diffusion. It is difficult to give any evidence on the structure of the defect clusters as no defects could be found by means of TEM analyses. Obviously, the defects are too small to be detected directly. Their presence, however, is clearly revealed by self-diffusion. This supports the stability of small defect clusters and thus theoretical calculations of the binding energy of $V$ and $I$ clusters as a function of the cluster size. $^{51}$

\section{CONCLUSION}

Experiments of self-diffusion in Si performed with an isotopically controlled ${ }^{28} \mathrm{Si} /{ }^{\text {nat }} \mathrm{Si} /{ }^{28} \mathrm{Si}$ sandwich structure grown by chemical vapor deposition and with a 20-bilayer structure of $\left({ }^{28} \mathrm{Si} /{ }^{29} \mathrm{Si}\right)_{20}$ grown by means of molecular beam epitaxy reveal a self-diffusion under thermal equilibrium and nonequilibrium conditions, respectively. The former experiments analyzed with SIMS confirm the low-temperature self-diffusion data reported by Shimizu et al. ${ }^{8}$ The latter experiments analyzed both with SIMS and NR demonstrate an enhancement of self-diffusion. Data obtained for equilibrium self-diffusion 
deviate from a single Arrhenius-type temperature dependence. Taking into account contributions of self-interstitials and vacancies to self-diffusion deduced from metal diffusion and constraints for the activation enthalpy of self-diffusion via vacancies deduced from dopant diffusion, the temperature dependence of self-diffusion under thermal equilibrium is most consistently described with temperature-dependent formation and migration enthalpies (entropies) assumed for vacancies. The temperature dependence of the vacancy properties describes a defect that gets more extended or spread out with increasing temperature. This concept was first proposed by Seeger and Chik $^{40}$ but could not be verified at that time due to experimental difficulties that have limited self-diffusion studies to a narrow temperature range. In the meantime, technical advances in epitaxial deposition and sputter profiling techniques have enabled self-diffusion experiments at low temperatures. The relevance of an extended vacancy can be independently verified by studying over a wide range of temperatures the behavior of dopants in $\mathrm{Si}$ whose diffusion is mainly mediated by vacancies. On the other hand, the enhanced self-diffusion in $\left({ }^{28} \mathrm{Si} /{ }^{29} \mathrm{Si}\right)_{20}$ multilayer structures is accurately modeled assuming self-interstitial- and vacancyrelated clusters that dissolve during annealing. Simulations of the experimental results reveal a binding energy of vacancies to clusters of $(3.2 \pm 0.2) \mathrm{eV}$ and a binding energy of self-interstitials to clusters of $(2.1 \pm 0.2) \mathrm{eV}$. The good agreement to experimental and theoretical results on the binding energy of defect clusters deduced from transient enhanced dopant diffusion in $\mathrm{Si}$ demonstrates the overall consistency of the presented interpretation of $\mathrm{Si}$ self-diffusion under equilibrium and nonequilibrium conditions.

\section{ACKNOWLEDGMENTS}

The authors thank Tascon GmbH Münster and RTG Mikroanalyse $\mathrm{GmbH}$ Berlin for SIMS measurements. This work was funded by the Deutsche Forschungsgemeinschaft under Grants No. BR 1520/12-1 and No. SCHM 1569/14-1 as well as an individual grant within the Heisenberg program for H.B. and H.S. The isotopically enriched $\mathrm{Si}$ was developed by the Initiatives for Proliferation Prevention Program of the Office of Nonproliferation Research and Engineering (NN-20) of the U. S. Department of Energy under Contract No. DEAC03-76SF00098. Neutron reflectometry experiments were performed at the Swiss spallation neutron source SINQ, Paul Scherrer Institute, Villigen, Switzerland, and have been also supported by the European Commission under the 7 th Framework Programme through the "Research Infrastructures" action of the "Capacities" Programme, Contract No CP-CSA-INFRA-2008-1.1.1 Number 226507-NMI3. *bracht@uni-muenster.de

${ }^{1}$ R. F. Peart, Phys. Status Solidi 15, K119 (1966).

${ }^{2}$ B. J. Masters and J. M. Fairfield, Appl. Phys. Lett. 8, 280 (1966).

${ }^{3}$ J. M. Fairfield and B. J. Masters, J. Appl. Phys. 38, 3148 (1967).

${ }^{4}$ R. N. Ghoshtagore, Phys. Rev. Lett. 16, 890 (1966).

${ }^{5}$ H. Bracht, E. E. Haller, and R. Clark-Phelps, Phys. Rev. Lett. 81, 393 (1998).

${ }^{6}$ A. Ural, P. B. Griffin, and J. D. Plummer, Phys. Rev. Lett. 83, 3454 (1999).

${ }^{7}$ S. R. Aid, T. Sakaguchi, K. Toyonaga, Y. Nakabayashi, S. Matumoto, M. Sakuraba, Y. Shimamune, Y. Hashiba, J. Murota,

K. Wada, and T. Abe, Mater. Sci. Eng., B 114-115, 330 (2004).

${ }^{8}$ Y. Shimizu, M. Uematsu, and K. M. Itoh, Phys. Rev. Lett. 98, 095901 (2007).

${ }^{9}$ H. Bracht, N. A. Stolwijk, and H. Mehrer, Phys. Rev. B 52, 16542 (1995).

${ }^{10}$ H. Bracht, Phys. Rev. B 75, 035210 (2007).

${ }^{11}$ H. Bracht, H. H. Silvestri, I. D. Sharp, and E. E. Haller, Phys. Rev. B 75, 035211 (2007).

${ }^{12}$ A. Ural, P. B. Griffin, and J. D. Plummer, Appl. Phys. Lett. 79, 4328 (2001).

${ }^{13}$ Y. Nakabayashi, H. I. Osman, T. Segawa, K. Saito, S. Matsumoto, J. Murota, K. Wada, and T. Abe, Jpn. J. Appl. Phys., Part 2 40, L181 (2001).

${ }^{14}$ Y. Nakabayashi, H. I. Osman, K. Yokota, K. Toyonaga, S. Matsumoto, J. Murota, K. Wada, and T. Abe, Mater. Sci. Semicond. Process. 6, 15 (2003).

${ }^{15}$ Y. Nakabayashi, H. I. Osman, K. Toyonaga, K. Yokota, S. Matsumoto, J. Murota, K. Wada, and T. Abe, Jpn. J. Appl. Phys. 42, 3304 (2003).
${ }^{16} \mathrm{~A}$ change in the correlation factor $f_{I}$ of self-diffusion via $I \mathrm{~s}$ affects the contribution $D_{\mathrm{Si}}^{I}$ to self-diffusion determined from metal-diffusion studies. The diffusion of $\mathrm{Au}$ and $\mathrm{Zn}$ in $\mathrm{Si}$ yields information about the uncorrelated contribution of $I$ to selfdiffusion, that is, the transport capacity $C_{I}^{\mathrm{eq}} D_{I} / C_{0}$, where $C_{I}^{\mathrm{eq}}$, $D_{I}$, and $C_{0}$ are the thermal equilibrium concentration of $I$, its diffusivity, and the $\mathrm{Si}$ atom density $\left(C_{0}=5 \times 10^{22} \mathrm{~cm}^{-3}\right)$, respectively. The $I$ contribution to self-diffusion is calculated by means of $D_{\mathrm{Si}}^{I}=f_{I} C_{I}^{\mathrm{eq}} D_{I} / C_{0}$ taking into account the transport capacity $C_{I}^{\mathrm{eq}} D_{I} / C_{0}$ and the correlation factor $f_{I} . f_{I}$ depends on the type of the specific $I$ involved in the self-diffusion process and on its diffusion pathways. Although different configurations of $I$ have been identified (see, e.g., Ref. 51 and references therein), the type of $I$ mediating self-diffusion remains unsolved and can also change with temperature.

${ }^{17}$ G. D. Watkins, Mater. Res. Soc. Symp. Proc. 469, 139 (1997).

${ }^{18}$ G. D. Watkins, J. Appl. Phys. 103, 106106 (2008).

${ }^{19}$ H. Bracht and A. Chroneos, J. Appl. Phys. 104, 076108 (2008).

${ }^{20}$ H. Bracht, E. E. Haller, K. Eberl, M. Cardona, and R. Clark-Phelps, Mater. Res. Soc. Symp. Proc. 527, 335 (1998).

${ }^{21}$ H. Bracht, Phys. B (Amsterdam) 376-377, 11 (2006).

${ }^{22}$ E. Hüger, R. Kube, H. Bracht, J. Stahn, T. Geue, and H. Schmidt, Phys. Status Solidi B 249, 2108 (2012).

${ }^{23}$ H. Schmidt, M. Gupta, and M. Bruns, Phys. Rev. Lett. 96, 055901 (2006).

${ }^{24}$ H. Schmidt, M. Gupta, J. Stahn, T. Gutberlet, and M. Bruns, Acta Mater. 56, 464 (2008).

${ }^{25}$ L. G. Parratt, Phys. Rev. 54, 359 (1954).

${ }^{26} \mathrm{C}$. Braun, PARRATT32 or the reflectometry tool, HMI, http://www.helmholtz-berlin.de 
${ }^{27}$ The self-diffusion data reported by Bracht et al. (Ref. 5), Shimizu et al. (Ref. 8), and those deduced from structure 2 were considered.

${ }^{28}$ K. Compaan and Y. Haven, Trans. Faraday Soc. 54, 1498 (1958).

${ }^{29}$ M. Posselt, F. Gao, and D. Zwicker, Phys. Rev. B 71, 245202 (2005).

${ }^{30}$ M. Posselt, F. Gao, and H. Bracht, Phys. Rev. B 78, 035208 (2008).

${ }^{31}$ A. Giese, H. Bracht, N. A. Stolwijk, and D. Baither, Mater. Sci. Eng. B 71, 160 (2000).

${ }^{32}$ N. A. Stolwijk, B. Schuster, and J. Hölzl, Appl. Phys. A 33, 133 (1984).

${ }^{33}$ N. A. Stolwijk, J. Hölzl, W. Frank, E. R. Weber, and H. Mehrer, Appl. Phys. A 39, 37 (1986).

${ }^{34}$ K. Compaan and Y. Haven, Trans. Faraday Soc. 52, 786 (1956).

${ }^{35}$ G. D. Watkins and J. W. Corbett, Phys. Rev. 138, A543 (1965).

${ }^{36}$ A. Bongiorno, L. Colombo, and T. Diaz De la Rubia, Europhys. Lett. 43, 695 (1998).

${ }^{37}$ P. Pochet and D. Caliste, Mater. Sci. Semicond. Process. 15, 675 (2012).

${ }^{38}$ A. Giese, H. Bracht, N. A. Stolwijk, and J. T. Walton, J. Appl. Phys. 83, 8062 (1998).

${ }^{39}$ A. Seeger, H. Föll, and W. Frank, in Radiation Effects in Semiconductors 1976, IOP Conf. Proc. No. 31 (Institute of Physics, London, 1977), p. 12.

${ }^{40}$ A. Seeger and K. P. Chik, Phys. Status Solidi 29, 455 (1968).

${ }^{41}$ P. Fahey, S. S. Iyer, and G. J. Scilla, Appl. Phys. Lett. 54, 843 (1989).

${ }^{42}$ A. N. Larsen and P. Kringhoj, Appl. Phys. Lett. 68, 2684 (1996).

${ }^{43}$ Self- and dopant diffusion in Ge under thermal equilibrium are mainly mediated by vacancies (see, e.g., Ref. 47). A contribution of self-interstitials in atomic transport in Ge under thermal equilibrium is not significant and can be neglected.

${ }^{44}$ S. Brotzmann and H. Bracht, J. Appl. Phys. 103, 033508 (2008).

${ }^{45}$ E. Hüger, U. Tietze, D. Lott, H. Bracht, D. Bougeard, E. E. Haller, and H. Schmidt, Appl. Phys. Lett. 93, 162104 (2008).

${ }^{46}$ M. Werner, H. Mehrer, and H. D. Hochheimer, Phys. Rev. B 32, 3930 (1985).

${ }^{47}$ S. Brotzmann, H. Bracht, J. L. Hansen, A. N. Larsen, E. Simoen, E. E. Haller, J. S. Christensen, and P. Werner, Phys. Rev. B. 77, 235207 (2008).
${ }^{48}$ M. Naganawa, Y. Shimizu, M. Uematsu, K. M. Itoh, K. Sawano, Y. Shiraki, and E. E. Haller, Appl. Phys. Lett. 93, 191905 (2008).

${ }^{49}$ A. Chroneos, H. Bracht, R. W. Grimes, and B. P. Uberuaga, Appl. Phys. Lett. 92, 172103 (2008).

${ }^{50}$ A. Chroneos, R. W. Grimes, B. P. Uberuaga, and H. Bracht, Phys. Rev. B. 77, 235208 (2008).

${ }^{51}$ L. Pelaz, L. A. Marqués, M. Aboy, P. López, and I. Santos, Eur. Phys. J. B 72, 323 (2009).

${ }^{52}$ A. Nylandsted Larsen, P. Kringhøj, J. Lundsgaard Hansen, and S. Y. Shiryaev, J. Appl. Phys. 81, 2173 (1997).

${ }^{53}$ Y. Zhao, M. J. Aziz, H.-J. Gossmann, S. Mitha, and D. Schiferl, Appl. Phys. Lett. 75, 941 (1999).

${ }^{54}$ A. Nylandsted Larsen, N. Zangenberg, and J. Fage-Pedersen, Mater. Sci. Eng. B 124-125, 241 (2005).

${ }^{55}$ D. Caliste and P. Pochet, Phys. Rev. Lett. 97, 135901 (2006).

${ }^{56}$ H. Bracht, J. F. Pedersen, N. Zangenberg, A. N. Larsen, E. E. Haller, G. Lulli, and M. Posselt, Phys. Rev. Lett. 91, 245502 (2003).

${ }^{57}$ P. A. Stolk, D. J. Eaglesham, H.-J. Gossmann, and J. M. Poate, Appl. Phys. Lett. 66, 1370 (1995).

${ }^{58}$ E. N. Sgourou, D. Timerkaeva, C. A. Londos, D. Aliprantis, A. Chroneos, D. Caliste, and P. Pochet, J. Appl. Phys. 113, 113506 (2013).

${ }^{59}$ N. E. B. Cowern, G. Mannino, P. A. Stolk, F. Roozeboom, H. G. A. Huizing, J. G. M. van Berkum, F. Cristiano, A. Claverie, and M. Jaraíz, Phys. Rev. Lett. 82, 4460 (1999).

${ }^{60}$ A. C. Damask and G. J. Dienes, Point Defects in Metals (Gordon and Breach, New York, 1963).

${ }^{61}$ H.-J. Gossmann, C. S. Rafferty, F. C. Unterwald, T. Boone, T. K. Mogi, M. O. Thompson, and H. S. Luftman, Appl. Phys. Lett. 67, 1558 (1995).

${ }^{62}$ H. Bracht, S. Schneider, J. N. Klug, C. Y. Liao, J. L. Hansen, E. E. Haller, A. N. Larsen, D. Bougeard, M. Posselt, and C. Wündisch, Phys. Rev. Lett. 103, 255501 (2009).

${ }^{63}$ S. Schneider, H. Bracht, J. N. Klug, J. L. Hansen, A. N. Larsen, D. Bougeard, and E. E. Haller, Phys. Rev. B 87, 115202 (2013).

${ }^{64}$ R. Kalyanaraman, T. E. Haynes, O. W. Holland, H.-J. L. Gossmann, C. S. Rafferty, and G. H. Gilmer, Appl. Phys. Lett. 79, 1983 (2001).

${ }^{65}$ V. C. Venezia, L. Pelaz, H.-J. L. Gossmann, T. E. Haynes, and C. S. Rafferty, Appl. Phys. Lett. 79, 1273 (2001). 